\title{
Bed forms of soft poroelastic material in an alluvial channel
}

\author{
P.C. Hsieh ${ }^{\text {a }}$, L.H. Huang ${ }^{\text {b,* }}$, T.W. Wang ${ }^{\text {b }}$ \\ ${ }^{a}$ Hydrotech Research Institute, National Taiwan University, Taipei 106, Taiwan, ROC \\ b Department of Civil Engineering and Hydraulic Research Laboratory, National Taiwan University, Taipei 106, Taiwan, ROC
}

Received 28 September 1999; in revised form 7 July 2000

\begin{abstract}
This study is to simulate stable bed forms of an alluvial channel with soft poroelastic bed caused by a constant current accompanied with water wave. Since a boundary layer exists within the soft porous bed near the homogeneouswater/porous-bed interface, conventional Stokes expansion, which only uses one parameter, $\varepsilon_{1}=k_{0} a$, fails to estimate the second longitudinal wave inside the soft poroelastic bed. In order to overcome this difficulty, a boundary layer correction approach applying Biot's theory of poroelasticity (J. Appl. Phys. 33 (4) (1962) 1482) for soft porous bed is proposed to simulate bed forms of dune, antidune, and flat bed by a two-parameter perturbation expansion based on $\varepsilon_{1}$ and $\varepsilon_{2}=k_{0} / k_{2}$ in the present study. A new Runge-Kutta/Newton-Raphson method to find wave numbers is also proposed, which can trace bed forms of different categories continuously, including dune, antidune and flat bed. Although we do not use an empirical sediment transport formula as Kennedy (J. Fluid. Mech. 16 (1963) 521) did, the present result not only confirms the stable dune and antidune of Kennedy (1963), but also finds a rapidly damping wave that Kennedy (1963) could not get. The dimensionless lagged distance $\operatorname{Re}\left(k_{0}\right) \delta$ in this study confirms Kennedy's (1963) comment and is found to be 0 , $\pi$, or $2 \pi$ for stable dune and antidune when the dissipative parameter, $\log \left(\operatorname{Im}\left(k_{0}\right) / \operatorname{Re}\left(k_{0}\right)\right)$, goes down. (c) 2001 Elsevier Science Ltd. All rights reserved.
\end{abstract}

Keywords: Boundary layer correction; Two-parameter perturbation; Nonlinear water wave

\section{Introduction}

In hydraulic engineering, the formation of bed form in an alluvial channel is one of the most concerned problems. Wave and current are both interrelated with bed form in an alluvial channel. Besides, the interaction between porous bed and homogeneous water flow is even more complicated. Therefore, stepby-step, studies of this field have been changed from linear flow to nonlinear flow and from rigid bed material to poroelastic bed material.

Putnam (1949) began the investigation on a linear water wave interacting with a porous bed. Then, Reid and Kajiura (1957) studied the porous bed problem by considering linear wave in an inviscid, incompressible and irrotational fluid flow satisfying Darcy's law interacting with a rigid, isotropic porous

\footnotetext{
${ }^{*}$ Corresponding author. Tel.: +886-2-2362-7588; fax: +886-2-2364-4513.

E-mail address: lhhwang@ce.ntu.edu.tw (L.H. Huang).
} 
skeleton. Sleath (1970) and Liu (1973) further improved the studies. However, all the aforementioned studies focused on the rigid bed material affected only by linear wave. As to nonlinear water wave problem, Mei (1983) and Fenton (1985) studied nonlinear wave in deep water on an impermeable rigid bed by Stokes expansion.

On the other hand, as for the bed form formation, Darwin (1883-1884) conducted experiments on sandripple caused by a oscillatory bed motion and concluded that the roller induced by a series of vortex acting on the sandy bed would probably render unstable ripple into stable state. Bagnold (1936) explained that the instability of dune of dessert was due to the impact of sand by wind. Exner (1925) established a differential erosion equation for two-dimensional flow, which indicated that the change in bed elevation was due to longitudinal variation of bottom velocity. Anderson (1953) applied Exner's (1925) erosion equation to explain the mechanism of the formation of ripple. However, the mechanism of dune and antidune is somehow different from that of ripple. Kennedy (1963) applied an empirical sediment transport formula to govern the continuity of the porous bed. And he used the instability analysis of potential theory to obtain his famous results of dune and antidune formations in alluvial channels. Unfortunately, owing to the constraint of instability analysis, Kennedy (1963) could only find the dominant wavelength of stable bed forms instead of the whole bed forms.

In fact, fluid within porous material interacting with deforming solid skeleton is a more complicated twophase problem for a realistic analysis. Biot (1956) developed theory of poroelasticity to discuss elastic wave in a fluid saturated porous solid. Mei and Foda (1981) proposed a boundary layer correction to simplify the analysis; however, their approach was without systematic perturbation analysis. Huang and Song (1993) solved the problem of oscillatory linear water waves interacting with a deformable bed by using three decoupled Helmoltz equations derived in Huang and Chwang (1990) to treat the bed as a poroelastic material. And in their solution, five nondimensional parameters were derived. Chen et al. (1997) also applied Huang and Chwang's (1990) approach and the conventional Stokes expansion of deep water wave based on $\varepsilon_{1}=k_{0} a$ to investigate the dynamic response of permeable bed material to nonlinear water waves. They found that the conventional Stokes expansion is only valid for hard poroelastic bed material but invalid for soft one even though the Ursell parameter is small. Huang and Chiang (1998) simulated stable bed forms under a constant current and linear oscillatory water waves by an approach similar to that in Huang and Song (1993). In the work of Huang and Chiang (1998), various bed forms of dune, antidune and flat bed under linear oscillatory water waves accompanied with a constant current were obtained. And the ambiguous lagged distance $\delta$ that has bothered researchers in river mechanics for many years was solved. Although a more clear hydraulic mechanism of formation of bed forms was found in the work of Huang and Chiang (1998), a much time-consuming method, however, was adopted.

For the stable bed form of a constant current flowing over a semi-infinite soft poroelastic deforming bed accompanied with nonlinear water waves (Fig. 1), a systematic two-parameter expansion based on $\varepsilon_{1}$ and $\varepsilon_{2}=k_{0} / k_{2}$ (Fig. 2), instead of the conventional one-parameter Stokes expansion based on $\varepsilon_{1}$, will be demonstrated in the present study. Moreover, a fast and automatically modified wave number solver combining Runge-Kutta method with Newton-Raphson method will be proposed.

\section{Formulation}

Fig. 1 indicates stable bed form formation of an alluvial channel caused by the plane waves propagating over a horizontal, infinitely thick and homogeneous poroelastic bed accompanied by a constant current. Region 1 is homogeneous water treated by potential theory while region 2 is a semi-infinite porous medium saturated with water simulated by the theory of poroelasticity by Biot (1962). The coordinate of region 1 ranges from $y=\xi^{*}(x, t)$ to $y=h+\eta^{*}(x, t)$, and that of region 2 from $y=\xi^{*}(x, t)$ to $y \rightarrow-\infty$. The symbols 


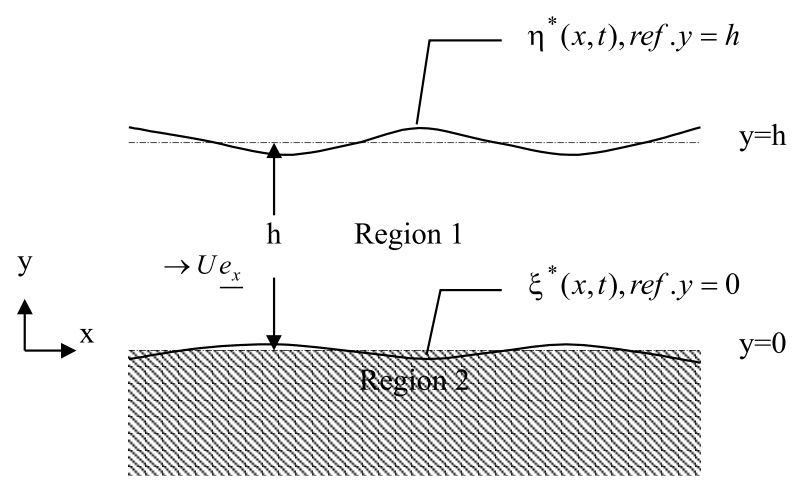

Fig. 1. Definition sketch.

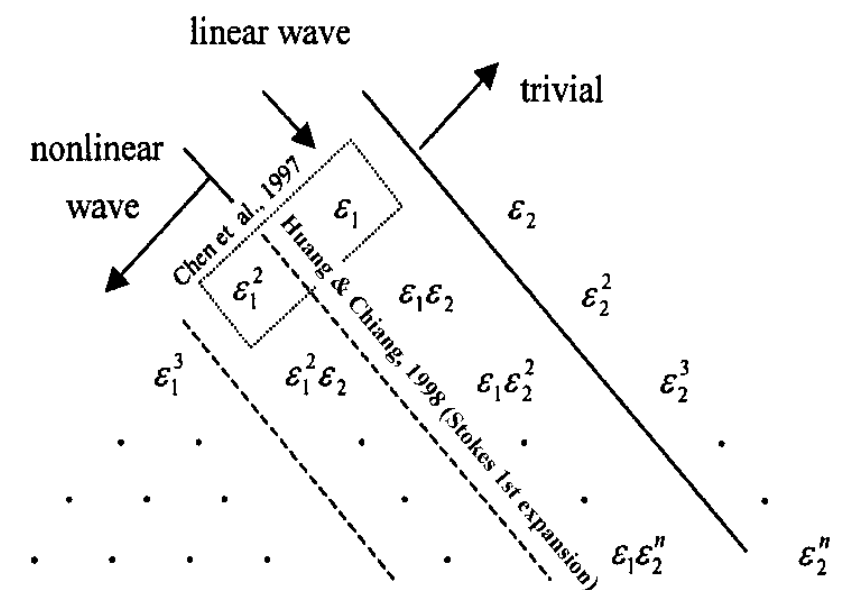

Fig. 2. Schematic diagram of the two-parameter expansion.

$\eta^{*}$ and $\xi^{*}$ represent the displacements of waves from the mean free surface $(y=h)$ and mean bed interface $(y=0)$, respectively.

\subsection{Boundary value problem}

Assuming that the homogeneous channel flow, region 1 of Fig. 1 , is potential flow, the velocity vector $\underline{u}_{1}^{*}$ can be represented by potential function $\Phi_{1}^{*}$ as

$$
\underline{u}_{1}^{*}=\nabla \Phi_{1}^{*} .
$$

Since the flow velocity may be written as a given constant current in the $x$ direction (i.e. $U \underline{e}_{x}$ ) plus a perturbed velocity $\underline{u}^{*(1)}$, thus,

$$
\underline{u}_{1}^{*}=U \underline{e}_{x}+\underline{u}^{*(1)} \text {. }
$$

By letting 


$$
\Phi_{1}^{*}=U x+\Phi^{*(1)},
$$

the following relation:

$$
\nabla \Phi^{*(1)}=\underline{u}^{*(1)}
$$

is satisfied.

The equations of continuity and momentum in terms of velocity potential $\Phi^{*(1)}$ become

$$
\begin{aligned}
& \nabla^{2} \Phi^{*(1)}=0 \\
& \rho_{0} \frac{\partial \Phi^{*(1)}}{\partial t}+\frac{\rho_{0}}{2}\left\{\left[U+\frac{\partial \Phi^{*(1)}}{\partial x}\right]^{2}+\left[\frac{\partial \Phi^{*(1)}}{\partial y}\right]^{2}\right\}+P^{*(1)}=0,
\end{aligned}
$$

where $P^{*(1)}$ is the perturbed pressure of homogeneous water, and $\rho_{0}$, the water density.

Referring to the work of Huang and Chwang (1990), the linear momentum equations of solid skeleton and fluid for the porous bed based on the theory of poroelasticity may be written as

$$
\begin{aligned}
& \nabla \cdot \underline{\underline{\sigma^{*}}}=\rho_{11} \frac{\partial^{2} \underline{d^{*}}}{\partial t^{2}}+\rho_{12} \frac{\partial^{2} \underline{D^{*}}}{\partial t^{2}}+b\left(\frac{\partial \underline{d^{*}}}{\partial t}-\frac{\partial \underline{D^{*}}}{\partial t}\right), \\
& \nabla \cdot \underline{\underline{S^{*}}}=\rho_{12} \frac{\partial^{2} \underline{d^{*}}}{\partial t^{2}}+\rho_{22} \frac{\partial^{2} \underline{D^{*}}}{\partial t^{2}}-b\left(\frac{\partial \underline{d}^{*}}{\partial t}-\frac{\partial \underline{D^{*}}}{\partial t}\right)
\end{aligned}
$$

with

$$
\begin{aligned}
& \underline{\underline{\sigma^{*}}}=\underline{\underline{\tau^{*}}}-\left(1-n_{0}\right) P^{*(2)} \underline{\underline{\mathrm{I}}}, \\
& \underline{\underline{\tau^{*}}}=2 G \underline{\underline{e^{*}}}+\lambda\left(\nabla \cdot \underline{d^{*}}\right) \underline{\underline{\mathrm{I}}}, \\
& \underline{\underline{e^{*}}}=\frac{1}{2}\left[\nabla \underline{d^{*}}+\left(\nabla \underline{d^{*}}\right)^{t}\right], \\
& \underline{\underline{S}}^{*}=-n_{0} P^{*(2)} \underline{\underline{\mathrm{I}}}, \\
& \rho_{11}=\left(1-n_{0}\right) \rho_{\mathrm{s}}+\rho_{a}, \\
& \rho_{12}=-\rho_{a}, \\
& \rho_{22}=n_{0} \rho_{0}+\rho_{a}, \\
& b=\mu n_{0}^{2} / k_{\mathrm{p}},
\end{aligned}
$$

where $\sigma^{*}$ is the solid stress tensor; $\tau^{*}$, the effective stress tensor of solid; $S^{*}$, the normal stress tensor of fluid; $\underline{d}^{*}$ and $\underline{\bar{D}}^{*}$, the solid and fluid displacement vectors, respectively; $P^{*(2)}$, the perturbed pressure of fluid inside the porous medium; $\rho_{\mathrm{s}}$, the solid density; $\rho_{a}$, the mass coupling effect (neglected in this study); $n_{0}$, the porosity; $\mu$, the fluid viscosity; $k_{\mathrm{p}}$, the specific permeability; $G$ and $\lambda$, Lame constants of elasticity; and $\underline{\mathbf{I}}$, the identity matrix.

Combining continuity equations of solid and fluid with equation of state of fluid and considering the slight compressibility of fluid inside the porous bed, the porosity of porous bed is expanded by perturbation and after linearization of the porosity, we can find 


$$
\frac{\partial P^{*(2)}}{\partial t}=-\frac{K}{n_{0}}\left[\left(1-n_{0}\right) \nabla \cdot\left(\frac{\partial d^{*}}{\partial t}\right)+n_{0} \nabla \cdot\left(\frac{\partial D^{*}}{\partial t}\right)\right]
$$

for perturbed pressure. In Eq. (17), $K$ is the bulk modulus of compressibility of fluid inside the porous bed.

The following three boundaries: (i) free surface $\left\lfloor y=h+\eta^{*}(x, t)\right\rfloor$, (ii) channel bed interface $\left\lfloor y=\xi^{*}(x, t)\right\rfloor$, and (iii) deep far field of porous bed $[y \rightarrow-\infty]$ must satisfy boundary conditions.

On the free surface, a kinematic boundary condition exists as

$$
-\frac{\partial \eta^{*}}{\partial x} \frac{\partial \Phi^{*(1)}}{\partial x}+\frac{\partial \Phi^{*(1)}}{\partial y}=\frac{\partial \eta^{*}}{\partial t}+U \frac{\partial \eta^{*}}{\partial x}
$$

and a dynamic boundary condition exists as

$$
\frac{\partial \Phi^{*(1)}}{\partial t}+\frac{1}{2}\left[\left(U+\frac{\partial \Phi^{*(1)}}{\partial x}\right)^{2}+\left(\frac{\partial \Phi^{*(1)}}{\partial y}\right)^{2}\right]+g \eta^{*}=0 .
$$

On the porous bed interface, the continuity of pressure gives

$$
P^{*(1)}=P^{*(2)},
$$

and the continuity of fluid flux gives

$$
\underline{n_{2}^{*}} \cdot\left[\left(1-n_{0}\right) \frac{\partial \underline{d}^{*}}{\partial t}+n_{0} \frac{\partial \underline{D}^{*}}{\partial t}\right]=\underline{n_{2}^{*}} \cdot\left(U \underline{e}_{x}+\nabla \Phi^{*(1)}\right),
$$

where

$$
\underline{n_{2}^{*}}=\left(\frac{-\partial \xi^{*}}{\partial x}, 1\right) / \sqrt{1+\left(\frac{\partial \xi^{*}}{\partial x}\right)^{2}}
$$

is the unit normal vector at the porous bed interface. Considering the kinematics of the porous bed interface, we have

$$
\frac{\partial \xi^{*}}{\partial t}=\frac{\partial d^{*}}{\partial t} \cdot\left(-\frac{\partial \xi^{*}}{\partial x}, 1\right)
$$

and Eq. (23) will be used to solve $\xi^{*}$. And the continuity of effective stresses of solid gives

$$
\underline{n_{2}^{*}} \cdot \underline{\underline{\tau^{*}}}=\underline{0} \text {. }
$$

At the far field of porous bed, $y \rightarrow-\infty$, the boundary conditions are vanishing displacement vectors, i.e.,

$$
\underline{d^{*}}, \underline{D^{*}} \rightarrow \underline{0} .
$$

If both $\left|\eta^{*}\right|$ and $\left|\xi^{*}\right|$ are much smaller than the relative wavelengths, it is more convenient to shift the boundary conditions at free surface, $y=h+\eta^{*}(x, t)$, and porous bed interface, $y=\xi^{*}(x, t)$, to $y=h$ and $y=0$ first before solving the boundary value problem. As conventionally, Taylor series expansions are applied to the boundary conditions at the free surface (18) and (19) and at the porous bed interface (20)(23) by performing

$$
\sum_{m=0}^{\infty} \frac{\left(\eta^{*}\right)^{m}}{m !} \frac{\partial^{m}}{\partial y^{m}}, \quad \sum_{m=0}^{\infty} \frac{\left(\xi^{*}\right)^{m}}{m !} \frac{\partial^{m}}{\partial y^{m}},
$$

respectively. 


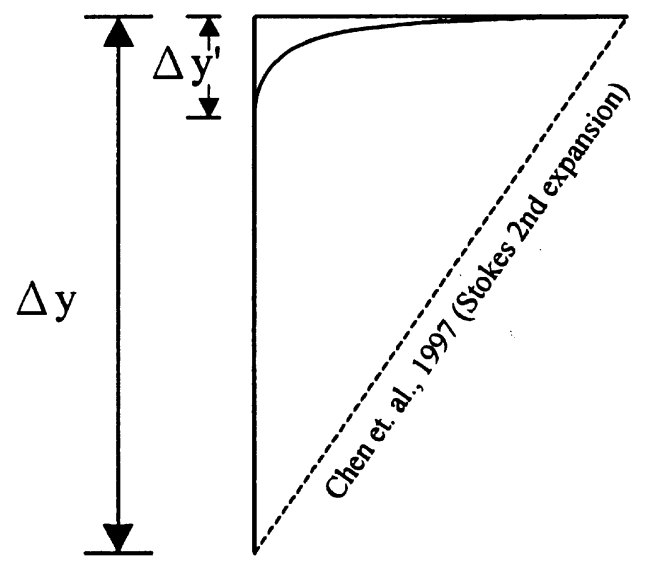

Fig. 3. Schematic diagram inside boundary layer for the second wave.

As $m=0$, the boundary value problem is linear, but as $m \geqslant 1$, the problem becomes nonlinear. For nonlinear problems, the above Taylor series expansions at the mean interface $(y=0)$ are applicable for the first and third waves, but not applicable for the second wave because there exists a boundary layer which will render errors of the partial derivative in the vertical direction for the second longitudinal wave. This is obviously indicated in Fig. 3. Fig. 3 shows that the length scale $\Delta y$ will overestimate the displacement potential of the second longitudinal wave because $\Delta y$ is much larger than $\Delta y^{\prime}$, and it is more accurate to evaluate the derivative by adopting the stretched length scale $\Delta y^{\prime}$. That's why Chen et al. (1997) failed to solve the nonlinear problem for soft porous material by only one length scale expansion. To overcome the difficulty, another small parameter, $\varepsilon_{2}=k_{0} / k_{2}$ other than $\varepsilon_{1}=k_{0} a$ needs to be proposed and furthermore, the vertical coordinate $y$ for the second wave will also be enlarged into $y^{\prime}$ based on this small parameter $\varepsilon_{2}$ (see Eq. (42b)).

Referring to the work of Huang and Song (1993) for the decoupling processes of Biot's equations of poroelasticity, the governing Eqs. (7) and (8) can be rewritten into three decoupled scalar equations as

$$
\nabla^{2} \Phi_{j}^{*(2)}+k_{j}^{2} \Phi_{j}^{*(2)}=0, \quad j=1,2,3 .
$$

Also, the perturbed pressure equation (17) gives

$$
P^{*(2)}=\frac{K}{n_{0}}\left[\left(1-n_{0}+\alpha_{1} n_{0}\right) k_{1}^{2} \Phi_{1}^{*(2)}+\left(1-n_{0}+\alpha_{2} n_{0}\right) k_{2}^{2} \Phi_{2}^{*(2)}\right],
$$

where the wave numbers, $k_{j}$, and the solid/fluid related parameters, $\alpha_{j}$, are given as Eqs. (8)-(20) in the work of Huang and Song (1993). In Eq. (26), $\Phi_{1}^{(2)}$ and $\Phi_{2}^{(2)}$ are the displacement potentials of the first and the second longitudinal waves, respectively; while $\Phi_{3}^{(2)}$ is the displacement potential of the third transverse wave, i.e.,

$$
\begin{aligned}
& \underline{d}^{*}=\nabla \Phi_{1}^{*(2)}+\nabla \Phi_{2}^{*(2)}+\nabla^{\wedge}\left(\Phi_{3}^{*(2)} \underline{e}_{Z}\right), \\
& \underline{D}^{*}=\alpha_{1} \nabla \Phi_{1}^{*(2)}+\alpha_{2} \nabla \Phi_{2}^{*(2)}+\alpha_{3} \nabla^{\wedge}\left(\Phi_{3}^{*(2)} \underline{e}_{Z}\right) .
\end{aligned}
$$

Note that governing Eqs. (5) and (26), pressure and effective stresses Eqs. (6), (27), and (10), together with boundary conditions (18)-(21), (24), and (25) form the complete boundary value problem of the present study. 


\subsection{Nondimensionalized governing equations and boundary conditions}

Huang and Song (1993) defined the following parameters:

$$
\begin{aligned}
& m=(2 G+\lambda) n_{0} / K, \\
& \varepsilon=n_{0} \rho_{0} \omega / b \\
& \Lambda^{2}=\frac{n_{0} \rho_{0}+\left(1-n_{0}\right) \rho_{\mathrm{s}}}{2 G+\lambda+\left(K / n_{0}\right)} \frac{\omega^{2}}{k_{0}^{2}}, \\
& \Pi^{2}=\frac{\mathrm{i}(m+1)}{m \varepsilon} \frac{\rho_{0}}{K} \frac{\omega^{2}}{k_{0}^{2}}, \\
& \Psi^{2}=\frac{n_{0} \rho_{0}+\left(1-n_{0}\right) \rho_{\mathrm{s}}}{G} \frac{\omega^{2}}{k_{0}^{2}},
\end{aligned}
$$

in their solution of linear water waves propagating over a poroelastic bed. In which, $\varepsilon$ is called the penetrability parameter; $\omega$, the frequency; and $m$, the stiffness ratio of solid and fluid. $\Lambda$ and $\Psi$ are only functions of water wave speed and material (fluid and solid skeleton) properties; while $\Pi$ is not only a function of the same variables for $\Lambda$ and $\Psi$ but also depends on the permeability of porous medium.

For low penetrability, i.e., $\|\varepsilon\| \ll 1$, Eqs. (32)-(34) could be simplified to

$$
\begin{aligned}
& \Lambda^{2} \fallingdotseq\left(k_{1} / k_{0}\right)^{2}, \\
& \Pi^{2} \fallingdotseq\left(k_{2} / k_{0}\right)^{2}, \\
& \Psi^{2} \fallingdotseq\left(k_{3} / k_{0}\right)^{2} .
\end{aligned}
$$

Moreover, for soft solid skeleton, $\left\|k_{2}\right\| \gg\left\|k_{0}\right\|$ and such that $\left\|\Pi^{2}\right\| \fallingdotseq\left\|k_{2}^{2} / k_{0}^{2}\right\| \gg 1$. Since $\left\|\Lambda^{2}\right\|$ is always smaller than $\left\|\Psi^{2}\right\|$, we can obtain

$$
\left\|\Lambda^{2}\right\|<\left\|\Psi^{2}\right\| \ll 1 \ll\left\|\Pi^{2}\right\| .
$$

Based on the above discussion, we herewith define

$$
\begin{gathered}
\varepsilon_{1}=k_{0} a, \\
\varepsilon_{2}=k_{0} / k_{2}
\end{gathered}
$$

for later use.

After the tedious but straightforward analysis of order of magnitude for each dependent variable by referring to Huang and Song's (1993) solution, the dimensionless variables are selected as

$$
\begin{aligned}
& \hat{x}=k_{0} x, \\
& \hat{y}=k_{0} y, \\
& y^{\prime}=\hat{y} / \varepsilon_{2}, \quad \text { for the second longitudinal wave only, } \\
& \hat{t}=\sqrt{g k_{0}} t,
\end{aligned}
$$




$$
\begin{aligned}
& \hat{\eta}^{*}=k_{0} \eta^{*}, \\
& \hat{\omega}=\omega / \sqrt{g k_{0}}, \\
& \hat{\Phi}^{*(1)}=\frac{k_{0}^{2}}{\sqrt{g k_{0}}} \Phi^{*(1)}, \\
& \hat{\Phi}_{1}^{*(2)}=\mathrm{e}^{k_{0} h} k_{0}^{2} \Phi_{1}^{*(2)}, \\
& \hat{\Phi}_{2}^{*(2)}=\mathrm{e}^{k_{0} h} k_{0}^{2} \frac{k_{2}^{2}}{k_{1}^{2}} \Phi_{2}^{*(2)}=\frac{\mathrm{e}^{k_{0} h} k_{0}^{2}}{\varepsilon_{2}^{2} \Lambda^{2}} \Phi_{2}^{*(2)}, \\
& \hat{\Phi}_{3}^{*(2)}=\mathrm{e}^{k_{0} h} k_{0}^{2} \Phi_{3}^{*(2)}, \\
& \hat{\xi}^{*}=\frac{k_{0} \mathrm{e}^{k_{0} h}}{\Psi^{2}} \xi^{*}, \\
& \hat{P}^{*(1)}=\frac{k_{0}}{\rho_{0} g} P^{*(1)}, \\
& \hat{P}^{*(2)}=\frac{k_{0}}{\rho_{0} g} P^{*(2)}, \\
& \hat{U}=\frac{k_{0}}{\sqrt{g k_{0}}} U .
\end{aligned}
$$

All the symbols of variables on the left-hand side of Eqs. (41)-(53) are dimensionless, but those on the righthand side are dimensional. Note that since multiple length scales in vertical direction are needed (Fig. 3 ), $\hat{y}$ and $y^{\prime}$ are proposed for the boundary layer correction approach.

Applying the two-parameter perturbation expansion, we can write the velocity potential of channel flow and the displacement potentials of the two kinds of wave for the whole domain as

$$
\begin{aligned}
& \hat{\Phi}^{*(1)}=\varepsilon_{1} \hat{\phi}_{10}^{*}+\varepsilon_{1} \varepsilon_{2} \hat{\phi}_{11}^{*}+\varepsilon_{1}^{2} \hat{\phi}_{20}^{*}+\mathrm{O}\left(\varepsilon_{1}^{2} \varepsilon_{2}, \ldots\right), \\
& \hat{\Phi}_{j}^{*(2)}=\varepsilon_{1} \hat{\phi}_{10}^{*[j]}+\varepsilon_{1} \varepsilon_{2} \hat{\phi}_{11}^{*[j]}+\varepsilon_{1}^{2} \hat{\phi}_{20}^{*[j]}+\mathrm{O}\left(\varepsilon_{1}^{2} \varepsilon_{2}, \ldots\right), \quad j=1,3 .
\end{aligned}
$$

Due to Eq. (38), the second wave needs to be solved inside the boundary layer. The displacement potential of the second wave inside the boundary layer is nondimensionalized specially as Eq. (48) and is expanded as

$$
\hat{\Phi}_{2}^{*(2)}=\varepsilon_{1} \hat{\phi}_{10}^{*[2]}+\varepsilon_{1} \varepsilon_{2} \hat{\phi}_{11}^{*[2]}+\varepsilon_{1}^{2} \hat{\phi}_{20}^{*[2]}+\mathrm{O}\left(\varepsilon_{1}^{2} \varepsilon_{2}, \ldots\right),
$$

if $\left\|\varepsilon_{2}\right\|$ and $\left\|\varepsilon_{1}\right\|$ are smaller than unity. Also, the profile of water wave at the free surface becomes

$$
\hat{\eta}^{*}=\varepsilon_{1} \hat{\eta}_{10}^{*}+\varepsilon_{1} \varepsilon_{2} \hat{\eta}_{11}^{*}+\varepsilon_{1}^{2} \hat{\eta}_{20}^{*}+\mathrm{O}\left(\varepsilon_{1}^{2} \varepsilon_{2}, \ldots\right),
$$

and that of the channel bed interface becomes

$$
\hat{\xi}^{*}=\varepsilon_{1} \hat{\xi}_{10}^{*}+\varepsilon_{1} \varepsilon_{2} \hat{\xi}_{11}^{*}+\varepsilon_{1}^{2} \hat{\xi}_{20}^{*}+\mathrm{O}\left(\varepsilon_{1}^{2} \varepsilon_{2}, \ldots\right) .
$$

For a periodic motion with the frequency $\omega$, the aforementioned variables []$^{*}(\underline{R}, t)$ can be written as []$(\underline{R}) \mathrm{e}^{-\mathrm{i} \omega t}$, where $\underline{R}$ is the position vector. Let the given incoming-wave amplitude before being disturbed by 
the porous bed be $a$ (i.e. $\hat{\eta}_{10}=\mathrm{e}^{\mathrm{i} \hat{x}}$ ) with a wave number $k_{0}$, and then Stokes expansion based on $\varepsilon_{1}$ and $\varepsilon_{2}$ will be carried out only to the first three terms for the present nonlinear water wave problem to avoid the occurrence of secular terms. Thus, after performing Taylor series expansions at the free surface and at the channel bed interface respectively, the boundary value problem of each order without the time factor is obtained in the following.

\subsection{Boundary value problem for the first and third waves}

\subsection{1. $O\left(\varepsilon_{1}\right)$}

The governing equations are

region (1): $-\infty<\hat{x}<\infty, 0<\hat{y}<k_{0} h$,

$$
\hat{\nabla}^{2} \hat{\phi}_{10}=0
$$

region (2): $-\infty<\hat{x}<\infty,-\infty<\hat{y}<0$,

$$
\begin{aligned}
& \hat{\nabla}^{2} \hat{\phi}_{10}^{[1]}+\Lambda^{2} \hat{\phi}_{10}^{[1]}=0, \\
& \hat{\nabla}^{2} \hat{\phi}_{10}^{[3]}+\Psi^{2} \hat{\phi}_{10}^{[3]}=0 .
\end{aligned}
$$

The boundary conditions are

at the free surface: $\hat{y}=k_{0} h,-\infty<\hat{x}<\infty$,

(a) kinematic free surface boundary condition

$$
\hat{\phi}_{10, \hat{y}}=-\mathrm{i} \hat{\omega} \hat{\eta}_{10}+\hat{U} \hat{\eta}_{10, \hat{x}},
$$

(b) dynamic free surface boundary condition

$$
-\mathrm{i} \hat{\omega} \hat{\phi}_{10}+\hat{U} \hat{\phi}_{10, \hat{x}}+\hat{\eta}_{10}=0 ;
$$

at the porous bed interface: $\hat{y}=0,-\infty<\hat{x}<\infty$,

(a) continuity of pressure

$$
-\mathrm{i} \hat{\omega} \hat{\phi}_{10}+\hat{U} \hat{\phi}_{10, \hat{x}}+\frac{k_{0} K \Lambda^{2}}{\mathrm{e}^{k_{0} h} n_{0} \rho_{0} g} q_{1} \hat{\phi}_{10}^{[1]}=0,
$$

(b) continuity of flux

$$
\begin{aligned}
& \mathrm{i} \hat{\omega} q_{1} \hat{\phi}_{10, \hat{y}}^{[1]}-\mathrm{i} \hat{\omega} q_{3} \phi_{10, \hat{x}}^{[3]}+\mathrm{e}^{k_{0} h} \hat{\phi}_{10, \hat{y}}=\hat{U}\left(\hat{\phi}_{10, \hat{x} \hat{y}}^{[1]}-\hat{\phi}_{10, \hat{x}}^{[3]}\right), \\
& \text { with } \\
& q_{j}=1-n_{0}+\alpha_{j} n_{0}, \quad j=1,3,
\end{aligned}
$$

(c) continuity of effective stress (only $\tau_{\hat{x} \hat{y}} \fallingdotseq 0$ )

$$
2 \hat{\phi}_{10, \hat{x} \hat{y}}^{[1]}+\hat{\phi}_{10, \hat{y} \hat{y}}^{[3]}-\hat{\phi}_{10, \hat{x} \hat{x}}^{[3]}=0 ;
$$

at the deep far field: $\hat{y} \rightarrow-\infty,-\infty<\hat{x}<\infty$,

$$
\hat{\phi}_{10}^{[j]} \rightarrow 0, \quad j=1,3 .
$$

Note that only one component of the above boundary condition of continuity of effective stress is needed, i.e. $\tau_{\hat{x} \hat{y}} \fallingdotseq 0$, otherwise it will become overdetermined. (Another condition, $\tau_{\hat{y} \hat{y}} \fallingdotseq 0$, includes the effect of the second wave and which will be adopted by the boundary layer correction for the second wave later.) 


\subsection{2. $O\left(\varepsilon_{1} \varepsilon_{2}\right)$}

The governing equations are

region (1): $-\infty<\hat{x}<\infty, 0<\hat{y}<k_{0} h$,

$$
\hat{\nabla}^{2} \hat{\phi}_{11}=0
$$

region (2): $-\infty<\hat{x}<\infty,-\infty<\hat{y}<0$,

$$
\begin{aligned}
& \hat{\nabla}^{2} \hat{\phi}_{11}^{[1]}+\Lambda^{2} \hat{\phi}_{11}^{[1]}=0, \\
& \hat{\nabla}^{2} \hat{\phi}_{11}^{[3]}+\Psi^{2} \hat{\phi}_{11}^{[3]}=0 .
\end{aligned}
$$

The boundary conditions are

at the free surface: $\hat{y}=k_{0} h,-\infty<\hat{x}<\infty$,

(a) kinematic free surface boundary condition

$$
\hat{\phi}_{11, \hat{y}}=-\mathbf{i} \hat{\omega} \hat{\eta}_{11}+\hat{U} \hat{\eta}_{11, \hat{x}}
$$

(b) dynamic free surface boundary condition

$$
\mathrm{i} \hat{\omega} \hat{\phi}_{11}=\hat{\eta}_{11}+\hat{U} \hat{\eta}_{11, \hat{x}} ;
$$

at the porous bed interface: $\hat{y}=0,-\infty<\hat{x}<\infty$,

(a) continuity of pressure

$$
-\mathrm{i} \hat{\omega} \hat{\phi}_{11}+\hat{U} \hat{\phi}_{11, \hat{x}}+\frac{k_{0} K \Lambda^{2}}{\mathrm{e}^{k_{0} h} n_{0} \rho_{0} g} q_{1} \hat{\phi}_{11}^{[1]}=0
$$

(b) continuity of flux

$$
\mathrm{i} \hat{\omega} q_{1} \hat{\phi}_{11, \hat{y}}^{[1]}-\mathrm{i} \hat{\omega} q_{3} \hat{\phi}_{11, \hat{x}}^{[3]}+\mathrm{e}^{k_{0} h} \hat{\phi}_{11, \hat{y}}=\hat{U}\left(\hat{\phi}_{11, \hat{y} \hat{y}}^{[1]}-\hat{\phi}_{11, \hat{x} \hat{x}}^{[3]}\right),
$$

(c) continuity of effective stress (only $\tau_{\hat{x} \hat{y}} \fallingdotseq 0$ )

$$
2 \hat{\phi}_{11, \hat{x} \hat{y}}^{[1]}+\hat{\phi}_{11, \hat{y} \hat{y}}^{[3]}-\hat{\phi}_{11, \hat{x} \hat{x}}^{[3]}=0 ;
$$

at the deep far field: $\hat{y} \rightarrow-\infty,-\infty<\hat{x}<\infty$,

$$
\hat{\phi}_{11}^{[j]} \rightarrow 0, \quad j=1,3 \text {. }
$$

Again, only one component of the boundary condition of continuity of effective stresses is needed, i.e. $\tau_{\hat{x} \hat{y}} \fallingdotseq 0$, for the same reason mentioned above.

\subsection{3. $O\left(\varepsilon_{1}^{2}\right)$}

The governing equations are

region (1): $-\infty<\hat{x}<\infty, 0<\hat{y}<k_{0} h$,

$$
\hat{\nabla}^{2} \hat{\phi}_{20}=0
$$

region (2): $-\infty<\hat{x}<\infty,-\infty<\hat{y}<0$,

$$
\begin{aligned}
& \hat{\nabla}^{2} \hat{\phi}_{20}^{[1]}+\frac{\tilde{k}_{1}^{2}}{k_{1}^{2}} \Lambda^{2} \hat{\phi}_{20}^{[1]}=0, \\
& \hat{\nabla}^{2} \hat{\phi}_{20}^{[3]}+\frac{\tilde{k}_{3}^{2}}{k_{3}^{2}} \Psi^{2} \hat{\phi}_{20}^{[3]}=0 .
\end{aligned}
$$

The boundary conditions are 
at the free surface: $\hat{y}=k_{0} h,-\infty<\hat{x}<\infty$,

(a) kinematic free surface boundary condition

$$
\hat{\phi}_{20, \hat{y}}+2 \mathrm{i} \hat{\omega} \hat{\eta}_{20}-\hat{U} \hat{\eta}_{20, \hat{x}}=\hat{\eta}_{10, \hat{x}} \hat{\phi}_{10, \hat{x}}-\hat{\eta}_{10} \hat{\phi}_{10, \hat{y} \hat{y}}
$$

(b) dynamic free surface boundary condition

$$
-2 \mathrm{i} \hat{\omega} \hat{\phi}_{20}+\hat{\eta}_{20}+\hat{U} \hat{\phi}_{20, \hat{x}}=\mathrm{i} \hat{\omega} \hat{\eta}_{10} \hat{\phi}_{10, \hat{y}}-\frac{1}{2}\left(\hat{\phi}_{10, \hat{x}}^{2}+\hat{\phi}_{10, \hat{y}}^{2}\right) ;
$$

at the porous bed interface: $\hat{y}=0,-\infty<\hat{x}<\infty$,

(a) continuity of pressure

$$
2 \mathrm{i} \hat{\omega} \hat{\phi}_{20}-\frac{k_{0} K \Lambda^{2}}{\mathrm{e}^{k_{0} h} n_{0} \rho_{0} g} \tilde{q}_{1} \hat{\phi}_{20}^{[1]}-\hat{U} \hat{\phi}_{20, \hat{x}}=\frac{1}{2}\left(\hat{\phi}_{10, \hat{x}}^{2}+\hat{\phi}_{10, \hat{y}}^{2}\right)+\mathrm{e}^{-k_{0} h} \Psi^{2} \hat{\xi}_{10}\left(\hat{U} \hat{\phi}_{10, \hat{x} \hat{y}}-\mathrm{i} \hat{\omega} \hat{\phi}_{10, \hat{y}}\right)+\frac{\Psi^{2} k_{0} K \Lambda^{2} \hat{\xi}_{10}}{\mathrm{e}^{2 k_{0} h} n_{0} \rho_{0} g} q_{1} \hat{\phi}_{10, \hat{y}}^{[1]}
$$

(b) continuity of flux

$$
\begin{aligned}
\mathrm{e}^{k_{0} h} \hat{\phi}_{20, \hat{y}}+2 \mathrm{i} \hat{\omega}\left(\tilde{q}_{1} \hat{\phi}_{20, \hat{y}}^{[1]}-\tilde{q}_{3} \hat{\phi}_{20, \hat{x}}^{[3]}\right)-\hat{U}\left(\hat{\phi}_{20, \hat{y}}^{[1]}-\hat{\phi}_{20, \hat{x}}^{[3]}\right)= & \Psi^{2} \hat{\xi}_{10, \hat{x}} \hat{\phi}_{10, \hat{x}}-\Psi^{2} \hat{\xi}_{10} \hat{\phi}_{10, \hat{y} \hat{y}}+\mathrm{i} \hat{\omega} \Psi^{2} \mathrm{e}^{-k_{0} h} \hat{\xi}_{10, \hat{x}}\left(q_{1} \hat{\phi}_{10, \hat{x}}^{[1]}+q_{3} \hat{\phi}_{10, \hat{y}}^{[3]}\right) \\
& -\mathrm{i} \hat{\omega} \Psi^{2} \mathrm{e}^{-k_{0} h} \hat{\xi}_{10}\left(q_{1} \hat{\phi}_{10, \hat{y} \hat{y}}^{[1]}-q_{3} \hat{\phi}_{10, \hat{x} \hat{x}}^{[3]}\right) \\
& +\hat{U} \Psi^{2} \mathrm{e}^{-k_{0} h} \hat{\xi}_{10}\left(\hat{\phi}_{10, \hat{x} \hat{y}}^{[1]}-\hat{\phi}_{10, \hat{x} \hat{y}}^{[3]}\right)
\end{aligned}
$$

(c) continuity of effective stress (only $\tau_{\hat{x} \hat{y}}=0$ )

$$
G\left(2 \hat{\phi}_{20, \hat{y} \hat{y}}^{[1]}+\hat{\phi}_{20, \hat{y} \hat{y}}^{[3]}-\hat{\phi}_{20, \hat{x} \hat{x}}^{[3]}\right)=\Psi^{2} \mathrm{e}^{-k_{0} h} \hat{\xi}_{10, \hat{x}}\left[2 G\left(\hat{\phi}_{10, \hat{x} \hat{x}}^{[1]}+\hat{\phi}_{10, \hat{y} \hat{y}}^{[3]}\right)-\lambda \Lambda^{2} \hat{\phi}_{10}^{[1]}-G \Psi^{2} \mathrm{e}^{-k_{0} h} \hat{\xi}_{10}\left(2 \hat{\phi}_{10, \hat{y} \hat{y}}^{[1]}+\hat{\phi}_{10, \hat{y} \hat{y} \hat{y}}^{[3]}-\hat{\phi}_{10, \hat{x} \hat{y}}^{[3]}\right)\right]
$$

at the deep far field: $\hat{y} \rightarrow-\infty,-\infty<\hat{x}<\infty$,

$$
\hat{\phi}_{20}^{[j]} \rightarrow 0, \quad j=1,3
$$

where $\tilde{k}_{j}$ and $\tilde{\alpha}_{j}(j=1,3)$ in nonlinear order $\varepsilon_{1}^{2}$ are given as Eqs. (8)-(20) in the work of Huang and Song (1993), and

$$
\tilde{q}_{j}=1-n_{0}+\tilde{\alpha}_{j} n_{0}, \quad j=1,3 .
$$

Again, only one component of the boundary condition of continuity of effective stresses is needed, i.e. $\tau_{\hat{x} \hat{y}}=0$, for the same reason mentioned above.

\subsection{Boundary layer correction for the second wave}

The second wave disappears outside the boundary layer but it does exist inside the boundary layer, so the complete solution needs to be corrected by further considering the second wave inside the porous material bed. When the perturbation expansion is expanded to the first two terms, the problem is only a linear problem. While it is expanded to the first three terms, the problem becomes a nonlinear problem. Since a thin boundary layer exists within the porous bed near the water/porous-bed interface, multiple length scales are necessary to solve the nonlinear boundary value problem for the second longitudinal wave (Fig. 3). We therefore let $y^{\prime}=\hat{y} / \varepsilon_{2}$ to change the scale from $\hat{y}$ to the magnified scale $y^{\prime}$ in Eq. (42b). The difficulty, the error due to the first partial derivative based on $y$ of the displacement potential of the second longitudinal wave, that Chen et al. (1997) encountered is now overcome by proposing two length scales in the vertical direction. After the coordinate transformation of Eq. (42b), the boundary value problem of the displacement potential of the second longitudinal wave inside the boundary layer is as follows. 


\subsection{1. $O\left(\varepsilon_{1}\right)$}

The governing equations are

$$
\hat{\phi}_{10, y^{\prime} y^{\prime}}^{[2]}+\hat{\phi}_{10}^{[2]}=0 .
$$

Boundary conditions: $y^{\prime}=0,-\infty<\hat{x}<\infty$,

(a) continuity of pressure

$$
\hat{\phi}_{10}^{[2]}=0,
$$

(b) continuity of effective stress (only $\tau_{\hat{y} \hat{y}} \fallingdotseq 0$ )

$$
2 G \Lambda^{2} \hat{\phi}_{10, y^{\prime} y^{\prime}}^{[2]}-\lambda \Lambda^{2} \hat{\phi}_{10}^{[2]}=\lambda \Lambda^{2} \hat{\phi}_{10}^{[1]}-2 G\left(\hat{\phi}_{10, \hat{y} \hat{y}}^{[1]}-\hat{\phi}_{10, \hat{y} \hat{y}}^{[3]}\right) .
$$

Note that the boundary condition of continuity of flux is equivalent to Eq. (89), and the boundary condition of $\tau_{\hat{x} \hat{y}} \fallingdotseq 0$ just satisfies $\hat{\phi}_{10, \hat{x} y^{\prime}}^{[2]}=-\hat{\phi}_{10, y^{\prime} \hat{x}}^{[2]}$, i.e. $\hat{\phi}_{10, \hat{x} y^{\prime}}^{[2]}=0$. However, $\hat{\phi}_{10, \hat{x} y^{\prime}}^{[2]}=0$ at $y^{\prime}=0$ is automatically satisfied by referring to Eq. (89). So only one component, $\tau_{\hat{y} \hat{y}}=0$, of the effective stress boundary conditions is needed to solve $\hat{\phi}_{10}^{[2]}$.

2.4.2. $O\left(\varepsilon_{1} \varepsilon_{2}\right)$

The governing equations are

$$
\hat{\phi}_{11, y^{\prime} y^{\prime}}^{[2]}+\hat{\phi}_{11}^{[2]}=0 .
$$

Boundary conditions: $y^{\prime}=0,-\infty<\hat{x}<\infty$,

(a) continuity of pressure

$$
\hat{\phi}_{11}^{[2]}=0,
$$

(b) continuity of effective stress (only $\tau_{\hat{y} \hat{y}} \fallingdotseq 0$ )

$$
2 G \Lambda^{2} \hat{\phi}_{11, y^{\prime} y^{\prime}}^{[2]}-\lambda \Lambda^{2} \hat{\phi}_{11}^{[2]}=\lambda \Lambda^{2} \hat{\phi}_{11}^{[1]}-2 G\left(\hat{\phi}_{11, \hat{y} \hat{y}}^{[1]}-\hat{\phi}_{11, \hat{x} \hat{y}}^{[3]}\right) .
$$

2.4.3. $O\left(\varepsilon_{1}^{2}\right)$

The governing equations are

$$
\hat{\phi}_{20, y^{\prime} y^{\prime}}^{[2]}+\hat{\phi}_{20}^{[2]}=0 .
$$

Boundary conditions: $y^{\prime}=0,-\infty<\hat{x}<\infty$,

(a) continuity of pressure

$$
\tilde{q}_{2} \hat{\phi}_{20}^{[2]}+\Psi^{2} \hat{\xi}_{10} \mathrm{e}^{-k_{0} h} q_{2} \hat{\phi}_{11, y^{\prime}}^{[2]}=0,
$$

(b) continuity of effective stress (only $\left.\tau_{\hat{y} \hat{y}} \fallingdotseq 0\right)$

$$
\begin{aligned}
2 G \Lambda^{2} \hat{\phi}_{20, y^{\prime} y^{\prime}}^{[2]}-\lambda \Lambda^{2} \hat{\phi}_{20}^{[2]}= & -2 G\left(\hat{\phi}_{20, \hat{y} \hat{y}}^{[1]}-\hat{\phi}_{20, \hat{y}}^{[3]}\right)+\lambda \Lambda^{2} \hat{\phi}_{20}^{[1]}-\Psi^{2} \hat{\xi}_{10} \mathrm{e}^{-k_{0} h}\left[2 G\left(\hat{\phi}_{10, \hat{y} \hat{y} \hat{y}}^{[1]}+\Lambda^{2} \hat{\phi}_{11, y^{\prime} y^{\prime} y^{\prime}}^{[2]}-\hat{\phi}_{10, \hat{x} \hat{y} \hat{y}}^{[3]}\right)\right. \\
& \left.-\lambda \Lambda^{2}\left(\hat{\phi}_{10, \hat{y}}^{[1]}+\hat{\phi}_{11, y^{\prime}}^{[2]}\right)\right] .
\end{aligned}
$$

The reasons for adopting only two boundary conditions in $\mathrm{O}\left(\varepsilon_{1} \varepsilon_{2}\right)$ and $\mathrm{O}\left(\varepsilon_{1}^{2}\right)$ are the same as those described in $\mathrm{O}\left(\varepsilon_{1}\right)$. 


\section{Analytical solution}

After the time factor $\mathrm{e}^{-\mathrm{i} \omega t}$ is omitted, the given incoming water wave profile with magnitude $a$ is

$$
\eta_{10}(x)=a \mathrm{e}^{\mathrm{i} k_{0} x} \quad(0<x<\infty) .
$$

With the input of incoming water wave, each order of the aforementioned boundary value problem can be solved in sequence as shown in Table 1 . Table 1 indicates that one must first find the solution of order $\varepsilon_{1}$ outside the boundary layer, and subsequently proceed to match with the inner expansion to complete the solution of order $\varepsilon_{1}$. Then, the solution of order $\varepsilon_{1}$ provides the possibility to solve the higher orders in sequence as indicated by the solid arrows as shown in Table 1 . Note that the problem of order $\varepsilon_{1} \varepsilon_{2}$ is solved simultaneously to find the unique solution. Thus, the dimensional solutions of the first longitudinal wave and the third transverse wave throughout the entire domain are obtained as follows.

\section{1. $O\left(\varepsilon_{1}\right)$}

$$
\begin{aligned}
\phi_{10} & =\frac{\mathrm{i}}{k_{0}^{2}}\left[\frac{g k_{0}}{k_{0} U-\omega} \cosh k_{0}(h-y)-\left(k_{0} U-\omega\right) \sinh k_{0}(h-y)\right] \mathrm{e}^{\mathrm{i} k_{0} x}, \\
\phi_{10}^{[1]} & =\frac{1}{\mathrm{e}^{k_{0} h} k_{0}^{2}} a_{1} \mathrm{e}^{K_{1} k_{0} y+\mathrm{i} k_{0} x}, \\
\phi_{10}^{[3]} & =\frac{1}{\mathrm{e}^{k_{0} h} k_{0}^{2}} a_{3} \mathrm{e}^{K_{3} k_{0} y+\mathrm{i} k_{0} x},
\end{aligned}
$$

with the dispersion relation of a complex wave number, $k_{0}$, as

$$
\left[g k_{0}+\frac{T_{2}}{g k_{0}}\left(k_{0} U-\omega\right)^{2}\right] \sinh \left(k_{0} h\right)=\left[T_{2}+\left(k_{0} U-\omega\right)^{2}\right] \cosh \left(k_{0} h\right),
$$

where

$$
T_{2}=\frac{n_{0} \rho_{0} g}{k_{0} K \Lambda^{2} q_{1}}\left(q_{1} \omega K_{1}+\mathrm{i} q_{3} \omega L_{1}-k_{0} U K_{1}-\mathrm{i} k_{0} U L_{1}\right)\left(k_{0} U-\omega\right) .
$$

Table 1

Relation among solutions of each order

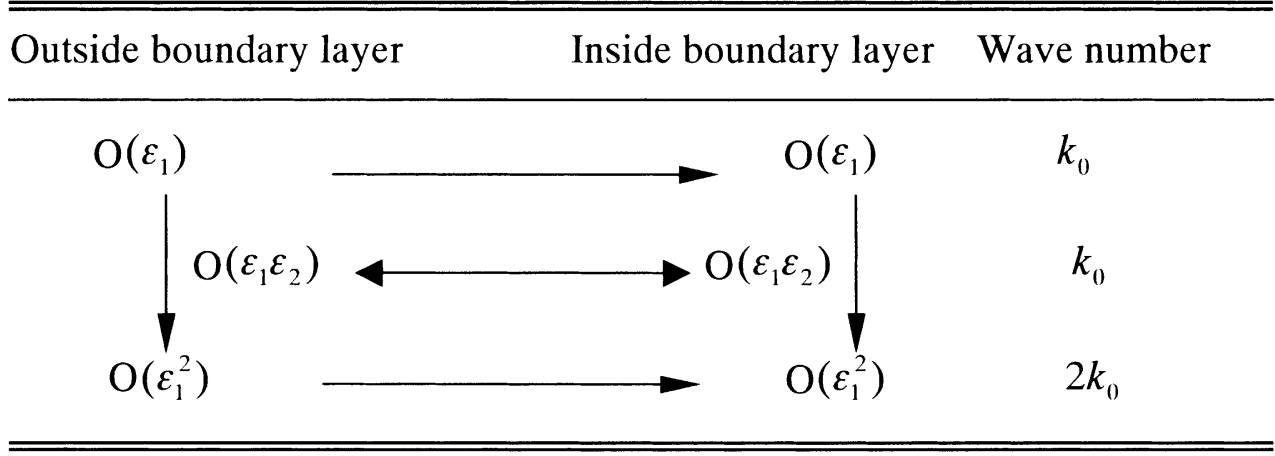


3.2. $O\left(\varepsilon_{1} \varepsilon_{2}\right)$

$$
\begin{aligned}
& \phi_{11}=\frac{\sqrt{g k_{0}}}{k_{0}^{2}} E_{5}\left[\cosh k_{0}(h-y)-\frac{\left(k_{0} U-\omega\right)^{2}}{g k_{0}} \sinh k_{0}(h-y)\right] \mathrm{e}^{\mathrm{i} k_{0} x}, \\
& \phi_{11}^{[1]}=\frac{1}{\mathrm{e}^{k_{0} h} k_{0}^{2}} c_{1} \mathrm{e}^{K_{1} k_{0} y+\mathrm{i} k_{0} x}, \\
& \phi_{11}^{[3]}=\frac{1}{\mathrm{e}^{k_{0} h} k_{0}^{2}} c_{3} \mathrm{e}^{K_{3} k_{0} y+\mathrm{i} k_{0} x}, \\
& \eta_{11}=\frac{\mathrm{i}}{k_{0} \sqrt{g k_{0}}}\left(\omega-k_{0} U\right) E_{5} \mathrm{e}^{\mathrm{i} k_{0} x} .
\end{aligned}
$$

3.3. $O\left(\varepsilon_{1}^{2}\right)$

$$
\begin{aligned}
& \phi_{20}=\frac{\sqrt{g k_{0}}}{k_{0}^{2}}\left[E_{3} \cosh 2 k_{0}(h-y)+E_{4} \sinh 2 k_{0}(h-y)\right] \mathrm{e}^{2 \mathrm{i} k_{0} x}, \\
& \phi_{20}^{[1]}=\frac{1}{\mathrm{e}^{k_{0} h} k_{0}^{2}} b_{1} \mathrm{e}^{M_{1} k_{0} y+2 \mathrm{i} k_{0} x}, \\
& \phi_{20}^{[3]}=\frac{1}{\mathrm{e}^{k_{0} h} k_{0}^{2}} b_{3} \mathrm{e}^{M_{3} k_{0} y+2 \mathrm{i} k_{0} x}, \\
& \eta_{20}=\frac{g}{k_{0} U-\omega}\left(\frac{1}{k_{0} U-\omega}+\frac{\mathrm{i} E_{4}}{\sqrt{g k_{0}}}\right) \mathrm{e}^{2 \mathrm{i} k_{0} x} .
\end{aligned}
$$

The aforementioned coefficients of the solutions in each order are listed in Appendix A.

The dimensional solutions of the second longitudinal wave obtained by the boundary layer correction approach are as follows.

$O\left(\varepsilon_{1}\right)$

$$
\phi_{10}^{[2]}=\frac{a_{2}}{k_{0}^{2} \mathrm{e}^{k_{0} h}} \frac{k_{1}^{2}}{k_{2}^{2}} \mathrm{e}^{\mathrm{i} k_{0}\left(x-\left(y / \varepsilon_{2}\right)\right)},
$$

with

$$
\begin{gathered}
a_{2}=\frac{2 G K_{1}^{2}-\lambda \Lambda^{2}}{(2 G+\lambda) \Lambda^{2}} a_{1}-\frac{2 \mathrm{i} G K_{3}}{(2 G+\lambda) \Lambda^{2}} a_{3} . \\
O\left(\varepsilon_{1} \varepsilon_{2}\right) \\
\phi_{11}^{[2]}=\frac{c_{2}}{k_{0}^{2} \mathrm{e}^{k_{0} h}} \frac{k_{1}^{2}}{k_{2}^{2}} \mathrm{e}^{\mathrm{i} k_{0}\left(x-\left(y / \varepsilon_{2}\right)\right)},
\end{gathered}
$$

with

$$
c_{2}=\frac{1}{(2 G+\lambda) \Lambda^{2}}\left[\left(2 G N_{1}^{2}-\lambda \Lambda^{2}\right) c_{1}-2 \mathrm{i} G N_{3} c_{3}\right] .
$$




$$
\begin{aligned}
& O\left(\varepsilon_{1}^{2}\right) \\
& \phi_{20}^{[2]}=\frac{b_{2}}{k_{0}^{2} \mathrm{e}^{k_{0} h}} \frac{k_{1}^{2}}{k_{2}^{2}} \mathrm{e}^{\mathrm{i} k_{0}\left(2 x-\left(y / \varepsilon_{2}\right)\right)},
\end{aligned}
$$

with

$$
\begin{gathered}
b_{2}=\frac{1}{(2 G+\lambda) \Lambda^{2}}\left\{\mathrm { e } ^ { - k _ { 0 } h } ( a _ { 1 } K _ { 1 } - \mathrm { i } a _ { 3 } ) \left[\left(2 G K_{1}^{3}-2 \mathrm{i} G K_{1}-\lambda \Lambda^{2} K_{1}\right) a_{1}-\mathrm{i} G\left(3 K_{3}^{2}+1\right) a_{3}\right.\right. \\
\left.\left.+\mathrm{i}(2 G+\lambda) \Lambda^{2} c_{2}\right]+\left(2 G M_{1}^{2}-\lambda \Lambda^{2}\right) b_{1}-4 \mathrm{i} G M_{3} b_{3}\right\} .
\end{gathered}
$$

After solving the displacement potentials, all the other variables can be obtained. The wave profile of porous bed from Eq. (23) gives

$$
\begin{aligned}
\xi(x)= & a\left(K_{1} a_{1}-\mathrm{i} a_{3}\right) \mathrm{e}^{\mathrm{i} k_{0} x-k_{0} h}+\varepsilon_{2} a \mathrm{e}^{\mathrm{i} k_{0} x-k_{0} h}\left(c_{1} K_{1}-\mathrm{i} a_{2} \Lambda^{2}-\mathrm{i} c_{3}\right) \\
& +\varepsilon_{1} \frac{a}{2} \mathrm{e}^{2 \mathrm{i} k_{0} x-2 k_{0} h}\left\{\left(K_{1} a_{1}-\mathrm{i} a_{3}\right)\left\lceil\left(K_{1}^{2}+1\right) a_{1}-a_{2} \Lambda^{2}-2 \mathrm{i} K_{3} a_{3}\right\rceil+2 b_{1} M_{1} \mathrm{e}^{k_{0} h}-4 \mathrm{i} b_{3} \mathrm{e}^{k_{0} h}\right\},
\end{aligned}
$$

and the free surface of homogeneous water is

$$
\eta(x)=a \mathrm{e}^{\mathrm{i} k_{0} x}+\frac{\mathrm{i} a \varepsilon_{2}}{\sqrt{g k_{0}}}\left(\omega-k_{0} U\right) E_{5} \mathrm{e}^{\mathrm{i} k_{0} x}+\frac{a \varepsilon_{1}}{k_{0} U-\omega}\left(\frac{g k_{0}}{k_{0} U-\omega}-\mathrm{i} E_{4} \sqrt{g k_{0}}\right) \mathrm{e}^{2 \mathrm{i} k_{0} x} .
$$

\subsection{Wave number solver by Runge-Kutta/Newton-Raphson method}

So far, the problem of solving the wave number from the dispersion relation has bothered researchers for many years due to the difficulty of convergence of the roots. As to the present work, the wave number is complex and thus it is more difficult to find the roots than that of traditional water wave. Since the formation of bed forms is related to the flow, the current effect should be certainly taken into account. If a current, $U$, is given, the corresponding wave number, $k_{0}$, could be found by solving the dispersion relation of Eq. (101). However, Huang and Chiang (1998) searched for the wave numbers by inputting arbitrarily chosen currents and obtained discrete results. It is not only time-consuming but also apt to improper categorizing. To avoid wrong grouping and save much time, the present study proposes a faster and smarter algorithm as shown in Fig. 4. The flow chart indicates that the wave numbers with given different currents could be traced out continuously by the Runge-Kutta method once an initial solution is found, and then the Newton-Raphson method is adopted to promote the accuracy of $k_{0}$ to fit the dispersion relation better. This algorithm has transformed the original dispersion equation into an ordinary differential equation by the following steps.

At first, rewrite Eq. (101) as a new function $f$,

$$
f=\left[g k_{0}+\frac{T_{2}}{g k_{0}}\left(k_{0} U-\omega\right)^{2}\right] \sinh \left(k_{0} h\right)-\left[T_{2}+\left(k_{0} U-\omega\right)^{2}\right] \cosh \left(k_{0} h\right)=0,
$$

and then take total derivative of Eq. (119), i.e.

$$
\mathrm{d} f=\frac{\partial f}{\partial k_{0}} \mathrm{~d} k_{0}+\frac{\partial f}{\partial U} \mathrm{~d} U=0 .
$$




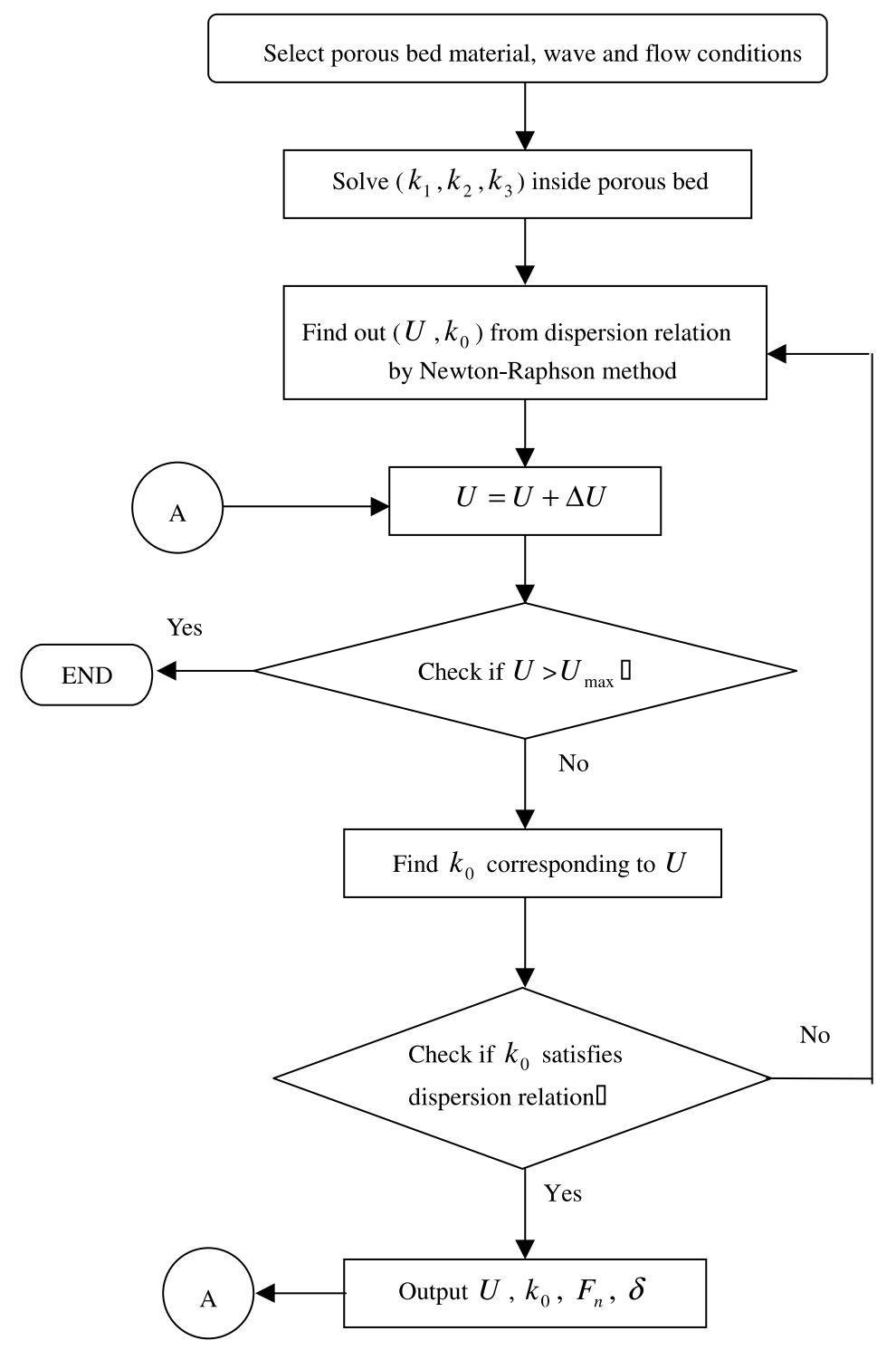

Fig. 4. Flow chart of wave number solver by Runge-Kutta/Newton-Raphson method.

Rewrite Eq. (120) as

$$
\frac{\mathrm{d} k_{0}}{\mathrm{~d} U}=-\frac{\frac{\partial f}{\partial U}}{\frac{\partial f}{\partial k_{0}}},
$$

where 


$$
\begin{aligned}
& \frac{\partial f}{\partial k_{0}}=\sinh \left(k_{0} h\right)\left(g-\left(c ( k _ { 0 } U - \omega ) ^ { 3 } \left(2 U k_{0}^{5} k_{3}^{2}+U k_{0}^{3} k_{3}^{2}\left(k_{3}^{2}-4 k_{1}^{2}\right)-\omega k_{1}^{2} k_{3}^{4} q_{1}+2 \omega k_{0}^{2} k_{1}^{2} k_{3}^{2}\left(2 q_{1}+q_{3}\right)\right.\right.\right. \\
& \left.\left.\left.-4 \omega k_{0}^{4}\left(k_{1}^{2}\left(q_{1}-q_{3}\right)+k_{3}^{2} q_{3}\right)\right)\right)\right) /\left(k_{0}^{3}\left(k_{3}^{2}-2 k_{0}^{2}\right)^{2} \sqrt{1-\frac{k_{1}^{2}}{k_{0}^{2}}}+3 c U\left(k_{0} U-\omega\right)^{2} t_{3}\right) \\
& -\cosh \left(k_{0} h\right)\left(2 U\left(k_{0} U-\omega\right)-\left(\operatorname { c g } ( k _ { 0 } U - \omega ) \left(2 U k_{0}^{5} k_{3}^{2}+U k_{0}^{3} k_{3}^{2}\left(k_{3}^{2}-4 k_{1}^{2}\right)-\omega k_{1}^{2} k_{3}^{4} q_{1}\right.\right.\right. \\
& \left.\left.\left.+2 \omega k_{0}^{2} k_{1}^{2} k_{3}^{2}\left(2 q_{1}+q_{3}\right)-4 \omega k_{0}^{4}\left(k_{1}^{2}\left(q_{1}-q_{3}\right)+k_{3}^{2} q_{3}\right)\right)\right)\right) /\left(\left(k_{0} k_{3}^{2}-2 k_{0}^{3}\right)^{2} \sqrt{1-\frac{k_{1}^{2}}{k_{0}^{2}}}\right. \\
& \left.+c g k_{0} U t_{3}+c g\left(k_{0} U-\omega\right) t_{3}\right)+h \cosh \left(k_{0} h\right)\left(g k_{0}+c\left(k_{0} U-\omega\right)^{3} t_{3}\right) \\
& +h \sinh \left(k_{0} h\right)\left(k_{0} U-\omega\right)\left(\omega-k_{0}\left(U+c g t_{3}\right)\right), \\
& \frac{\partial f}{\partial U}=k_{0}\left(-2 \cosh \left(k_{0} h\right)\left(k_{0} U-\omega\right)+\frac{1}{2 k_{0}^{2}-k_{3}^{2}}\left(c \sqrt { 1 - \frac { k _ { 1 } ^ { 2 } } { k _ { 0 } ^ { 2 } } } \left(\operatorname { s i n h } ( k _ { 0 } h ) ( k _ { 0 } U - \omega ) ^ { 2 } \left(6 \omega k_{0}^{2}\left(q_{1}-q_{3}\right)\right.\right.\right.\right. \\
& \left.\left.\left.\left.-k_{3}^{2}\left(\omega-4 k_{0} U+3 \omega q_{1}\right)\right)+g k_{0} \cosh \left(k_{0} h\right)\left(k_{3}^{2}\left(\omega-2 k_{0} U+\omega q_{1}\right)+2 \omega k_{0}^{2}\left(q_{3}-q_{1}\right)\right)\right)\right)\right) \text {, }
\end{aligned}
$$

with

$$
\begin{aligned}
c & =\frac{n_{0} \rho_{0}}{K k_{1}^{2} q_{1}}, \\
t_{3} & =\frac{\sqrt{1-\frac{k_{1}^{2}}{k_{0}^{2}}}\left(U k_{0} k_{3}^{2}-\omega k_{3}^{2} q_{1}+2 \omega k_{0}^{2}\left(q_{1}-q_{3}\right)\right)}{2 k_{0}^{2}-k_{3}^{2}} .
\end{aligned}
$$

Now Eq. (121) is an initial value problem, and it is solved by the fourth-order Runge-Kutta method (Press et al., 1992) in this study. Moreover, since this problem has been transformed into an initial value problem, all the other solutions could be guaranteed of the same family with a given initial value. In other words, all the solutions of the same line stand for the same kind of bed forms under different flow conditions.

\section{Results and comments}

Since the solution of Chen et al. (1997), which adopted a one-parameter perturbation for soft porous bed, failed due to the existence of a boundary layer, and the second longitudinal wave decays very quickly in the vertical direction near the interface, a two-parameter expansion based on $\varepsilon_{1}=k_{0} a$ and $\varepsilon_{2}=k_{0} / k_{2}$ needs to be proposed for the present work. Especially inside the boundary layer, the correction of the second wave is reformulated to complete the boundary value problem. However, this work is constrained to $\left\|\varepsilon_{1}\right\|^{1 / 2}>\left\|\varepsilon_{2}\right\|>\left\|\varepsilon_{1}\right\|^{2}$ before liquefaction by comparing layer 2 with layer 3 of Fig. 2.

From Eqs. (117) and (118), the profiles of free surface and channel bed are very dependent on both the flow and the material. Therefore the analysis of the bed forms by Kennedy (1963) is not complete due to considering flow conditions only but without concerning bed material. To confirm the validity of the proposed solution, the material property of a soft porous bed in Table 2 is selected, and Fig. 5 provides the 
Table 2

Flow conditions and property of soft porous bed material

\begin{tabular}{llll}
\hline Items & Notations & Values & Units \\
\hline (a) Water & & & \\
Density & $\rho_{0}$ & 1000 & $\mathrm{~kg} / \mathrm{m}^{3}$ \\
Bulk modulus & $K$ & $2.3 \times 10^{9}$ & $\mathrm{~N} / \mathrm{m}^{2}$ \\
Dynamic viscosity & $\mu$ & 0.001 & $\mathrm{Ns} / \mathrm{m}^{2}$ \\
Depth & $h$ & 2.0 & $\mathrm{~m}$ \\
Wave amplitude & $a$ & 0.2 & $\mathrm{~m}$ \\
Period & $T$ & 2.0 & $\mathrm{~s}$ \\
Current & $U$ & $0.01-15$ & $\mathrm{~m} / \mathrm{s}$ \\
(b) Skeleton & & & \\
Density & $\rho_{\mathrm{s}}$ & 2650 & $\mathrm{~kg} / \mathrm{m}^{3}$ \\
Lame's constant & $G$ & $5.0 \times 10^{4}$ & $\mathrm{~N} / \mathrm{m}^{2}$ \\
Lame's constant & $\lambda$ & $1.0 \times 10^{5}$ & $\mathrm{~N} / \mathrm{m}^{2}$ \\
Specific permeability & $k_{\mathrm{p}}$ & $1.0 \times 10^{-15}$ & $\mathrm{~m}$ \\
Porosity & $n_{0}$ & 0.4 & \\
\hline
\end{tabular}

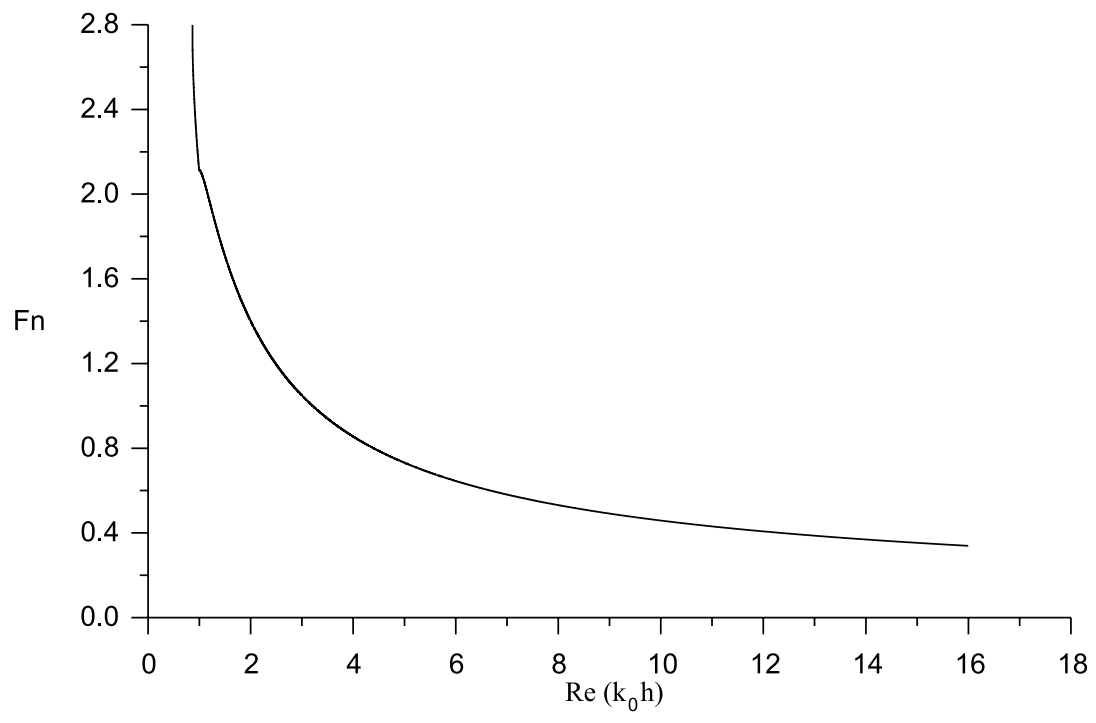

Fig. 5. Bed form envelope of Froude number versus dimensionless wave number (wave period $=2 \mathrm{~s}$ ).

bed form simulation result of Froude number $\left(F_{n}=U / \sqrt{g h}\right)$ versus dimensionless wave number $\left(\operatorname{Re}\left(k_{0} h\right)\right)$. The line in Fig. 5 delineates a bed form envelope curve under a given soft material and different currents, which means the maximum possible Froude number for the occurrence of long-crested features, as that in Fig. 9 of Kennedy (1963).

To predict various formations of bed forms, the same porous bed material in Table 2 is adopted to simulate the bed form category of dissipative parameter $\left(\log \left(\operatorname{Im}\left(k_{0}\right) / \operatorname{Re}\left(k_{0}\right)\right)\right)$ versus dimensionless lagged distance $\left(\operatorname{Re}\left(k_{0}\right) \delta / \pi\right)$ as shown in Fig. 6. In which, $\delta$ is an ambiguous lagged distance of sediment discharge from flow velocity suggested by Kennedy (1963). And, it just equals the phase difference between $\omega \xi^{*} / k_{0}$ from Eq. (117) and the perturbed velocity component $\underline{u}_{x}^{*(1)}$ from Eq. (4). From Kennedy's (1963) instability 


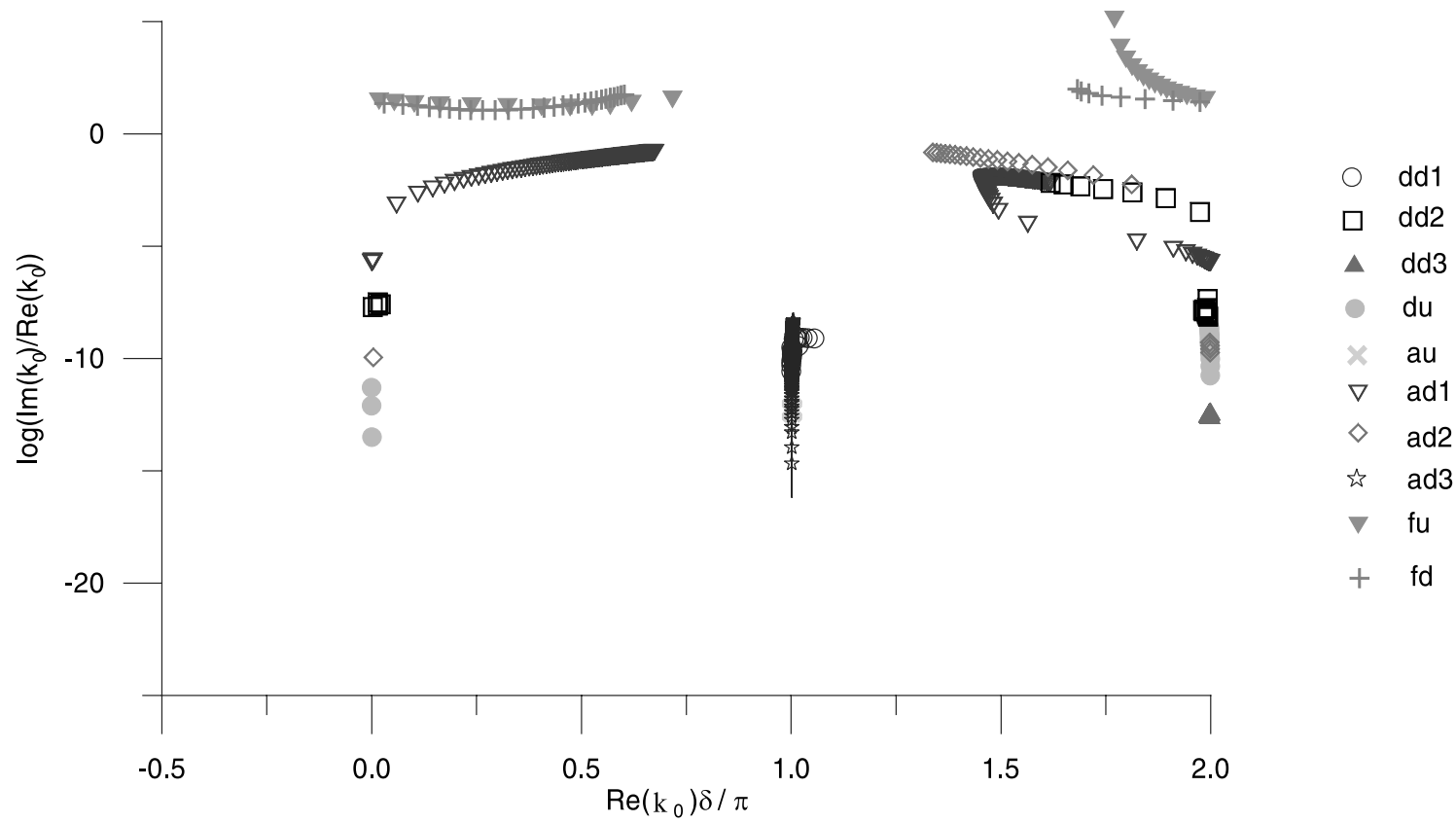

Fig. 6. Dissipative parameter versus dimensionless lagged distance (wave period $=2 \mathrm{~s}$ and depth $=2 \mathrm{~m}$ ).

analysis, it was found that only when $\operatorname{Re}\left(k_{0}\right) \delta / \pi$ equals 0 and 1 , there are stable bed forms; otherwise the bed forms are "unstable". Fig. 6 indicates that there are several bed forms to be found out including dune moving downstream (dd1, dd2 and dd3), dune moving upstream (du), antidune moving downstream (ad1 and $\mathrm{ad} 2$ ), antidune moving upstream (au), rapidly damping wave (i.e. flat bed) moving upstream (fu), and rapidly damping wave moving downstream (fd). It is noticeable that the solutions of the same kind of bed form are on the same line, which is continuously traced out by the solver as shown in Fig. 4, and the rapidly damping waves are defined to dissipate very quick, say the imaginary part of the wave number is larger than the real part of the wave number by ten or even more. So, the flat bed exists at larger dissipative parameters but the stable dune and antidune exist at smaller dissipative parameters. The dimensionless lagged distance $\operatorname{Re}\left(k_{0}\right) \delta$ in this study is found to be $0, \pi$, or $2 \pi$ for stable dune and antidune when the dissipative parameter goes down. The results not only confirm Kennedy's (1963) conclusion about $\delta$ for stable bed forms, but also provide correct information of rapidly damping bed forms (instead of "unstable" bed forms that grow to infinity given by Kennedy's (1963) analysis) such as flat bed and some rapidly damping dune and antidune that Kennedy (1963) did not get.

For further study, some physical quantities are illustrated to show the current effect $(U=0,0.1,0.5$ and $1.0 \mathrm{~m} / \mathrm{s}$ ) and the simulation results are compared in Figs. 7-10. Fig. 7 shows the pore pressure under four different currents. The pore pressure is getting smaller when the current effect is getting stronger. $P_{0}$ in Fig. 7 is the perturbed pressure on bed at $y=0$. In Fig. 8, near the interface $\left(y=\xi^{*}(x, t)\right)$, the effective stresses $\tau_{x x}$ and $\tau_{y y}$ become larger due to larger dilatation for stronger currents, and $\tau_{x y}$ is very close to zero, but it becomes larger for larger currents due to the stronger velocity gradients. In Fig. 9, the displacements of solid and fluid fluctuate more drastically for larger currents. Fig. 10 shows the profiles of channel bed and free surface. In Fig. 10(a), the free surface profiles are unchanged under different currents. This is because that the amplitudes of given waves are very small in the example. While in Fig. 10(b), the channel bed interface profiles fluctuate as the displacements of solid of Fig. 9 do. Therefore the present solution not only 

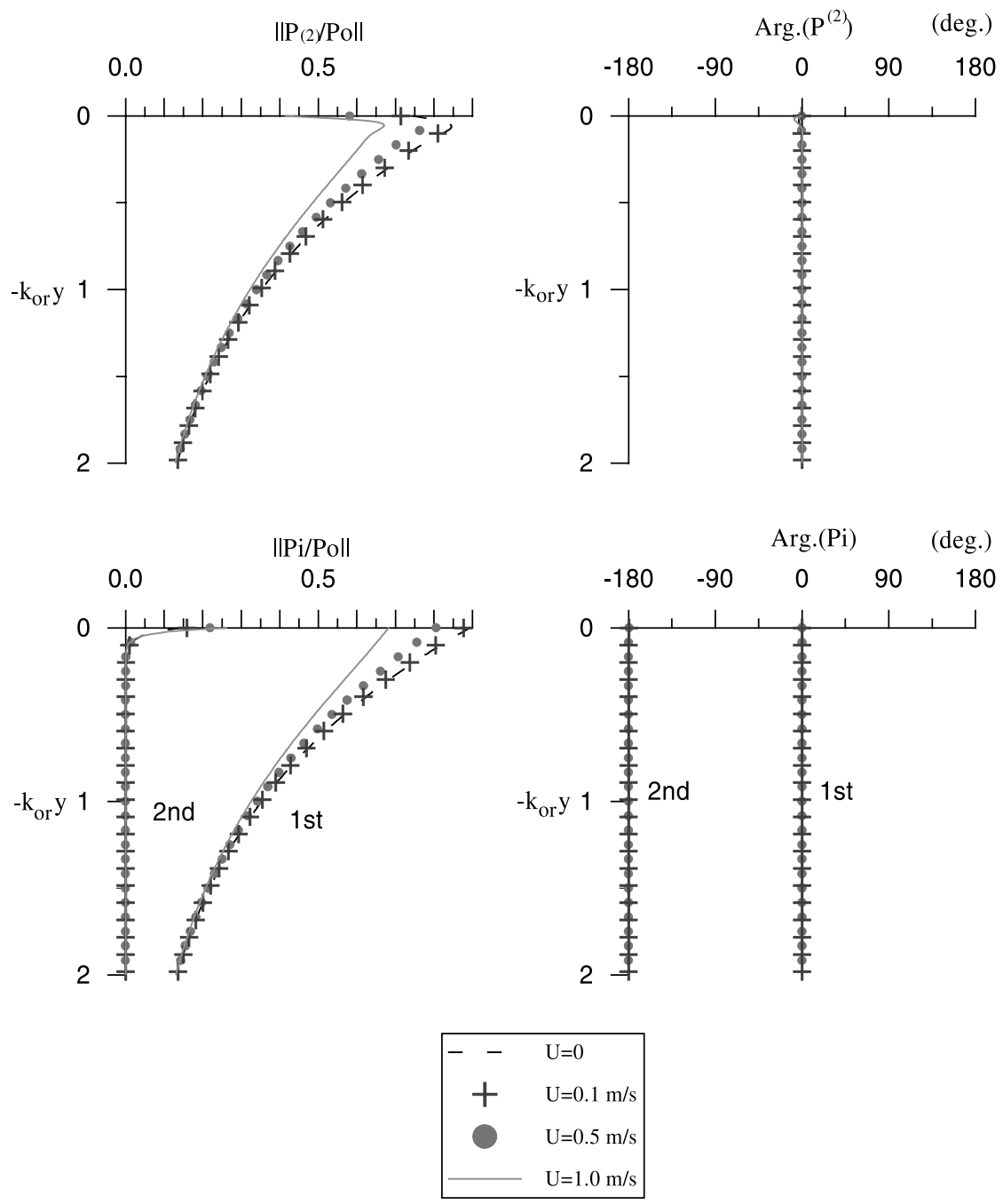

Fig. 7. Variation of pore pressures under different currents.

gives the formation mechanics of bed forms under the nonlinear oscillatory water waves and the current effect, but also shows the validity of Biot theory for a soft porous bed.

\section{Conclusions}

Since for a soft poroelastic bed there exists a boundary layer inside the porous bed and the wavelength of the second longitudinal wave is much shorter than that of the water wave, higher order Stokes expansion of water wave based on $\varepsilon_{1}=k_{0} a$ is invalid, i.e. one-parameter perturbation failed (Chen et al., 1997). Therefore, considering the second length scale based on the second longitudinal wave is necessary, and thus 

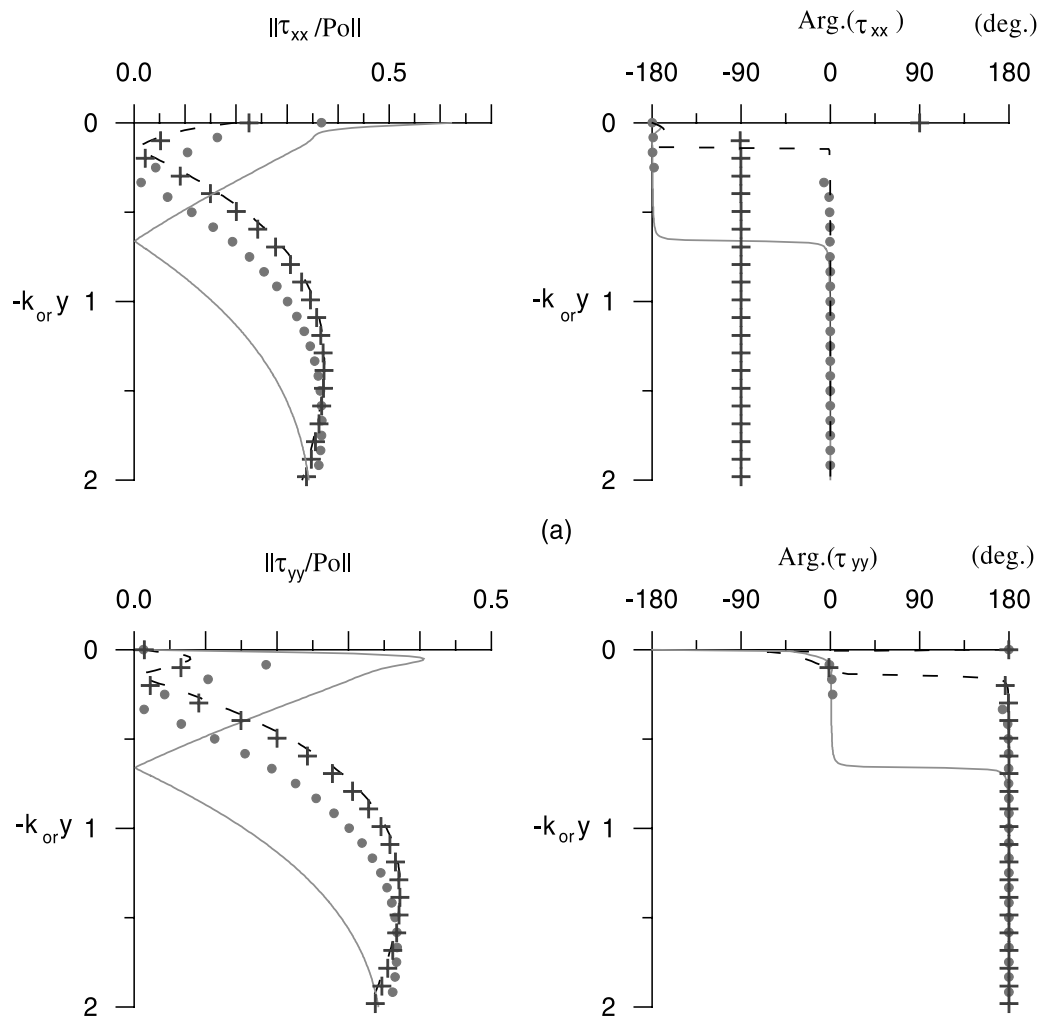

(a)
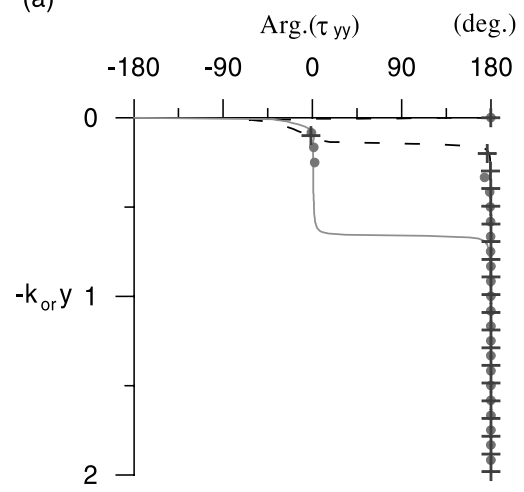

(b)

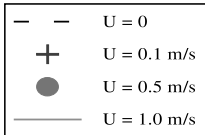

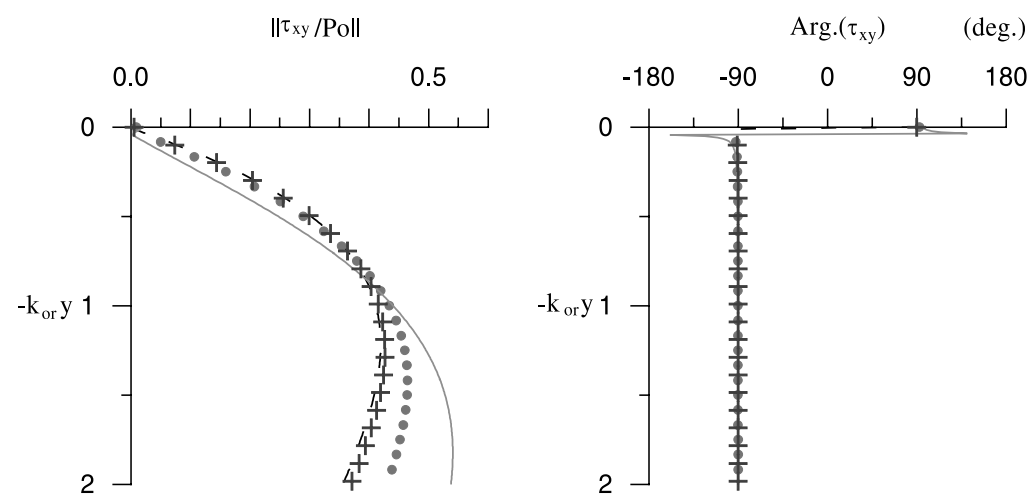

(c)

$$
\begin{aligned}
-\quad \mathrm{U}=0 \\
+\quad \mathrm{U}=0.1 \mathrm{~m} / \mathrm{s} \\
\mathrm{U}=0.5 \mathrm{~m} / \mathrm{s} \\
\mathrm{U}=1.0 \mathrm{~m} / \mathrm{s}
\end{aligned}
$$

Fig. 8. Variation of effective stresses under different currents (a) $x x$, (b) $y y$ and (c) $x y$ component. 

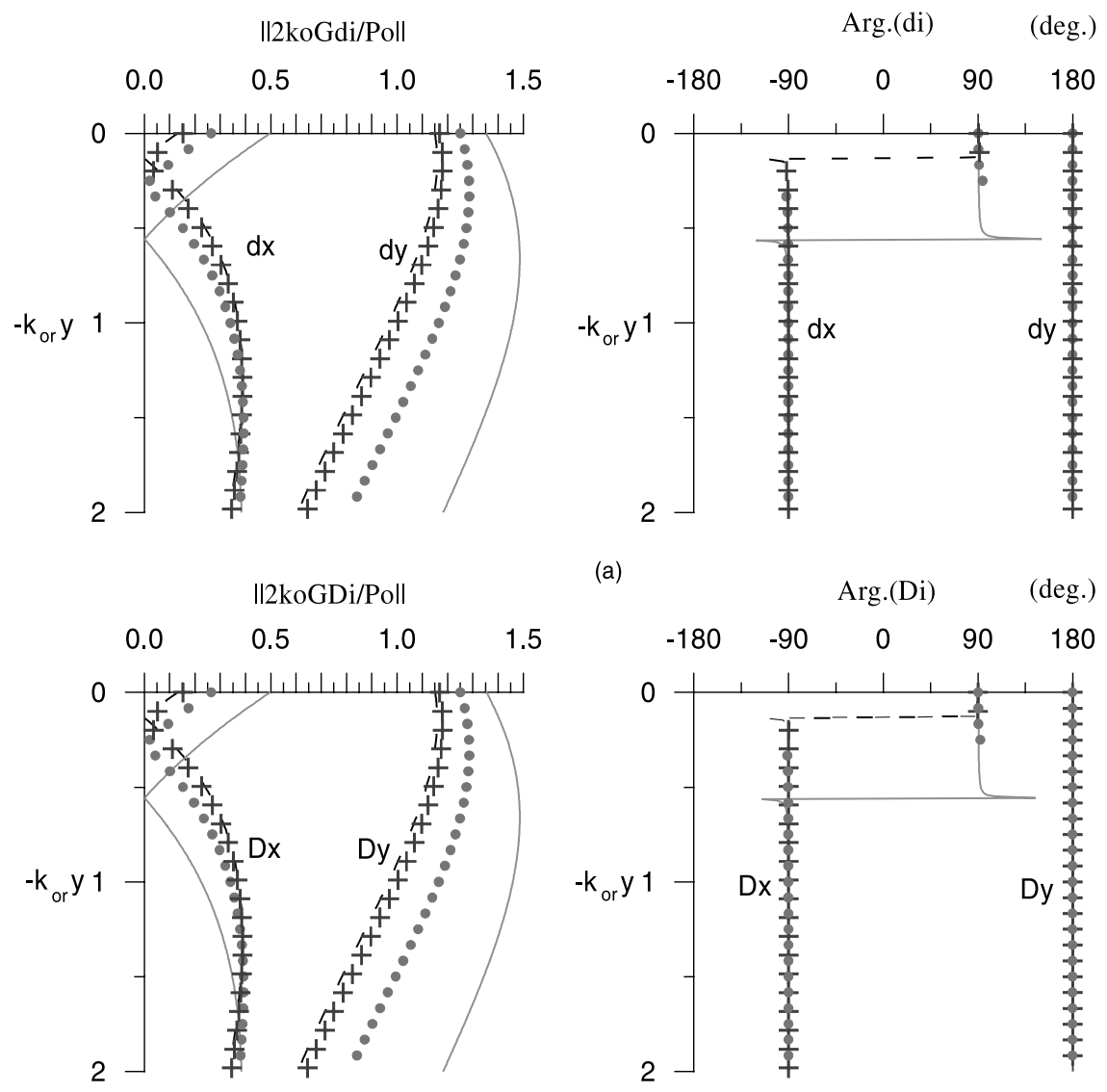

(a) Arg.(Di) (deg.)

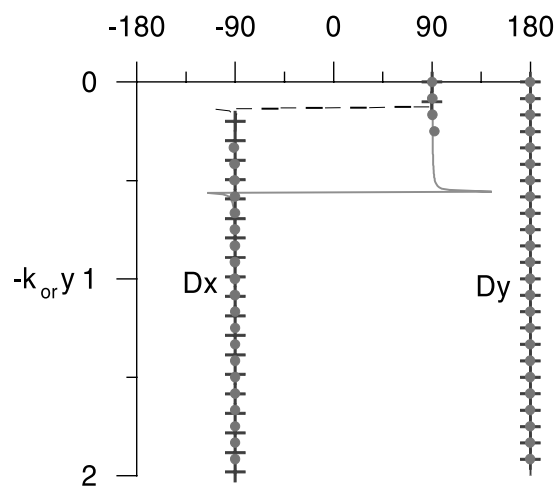

(b)

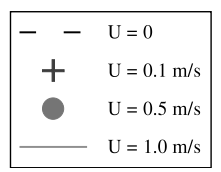

Fig. 9. Variation of displacements under different currents.

a two-parameter perturbation expansion based on $\varepsilon_{1}=k_{0} a$ and $\varepsilon_{2}=k_{0} / k_{2}$ is proposed. In addition, the second wave vanishes outside the boundary layer but exists inside it, so the complete solution of the displacement potential needs to be corrected inside the boundary layer. The complete boundary-valueproblem is solved systematically by the boundary layer correction approach in the present study.

In spite of different approaches, the present work not only confirms the stable dune and antidune of Kennedy (1963), but also finds rapidly damping waves that Kennedy (1963) could not get. Moreover, the present study proposes a solver to continuously trace out the wave numbers of the same kind of bed forms and gets higher accuracy by adopting a new Runge-Kutta/Newton-Raphson method. Finally, the dimensionless lagged distance $\operatorname{Re}\left(k_{0}\right) \delta$ confirms Kennedy's (1963) comment and is found to be $0, \pi$, or $2 \pi$ for stable dune and antidune while the dissipative parameter goes down as shown in Fig. 6. 


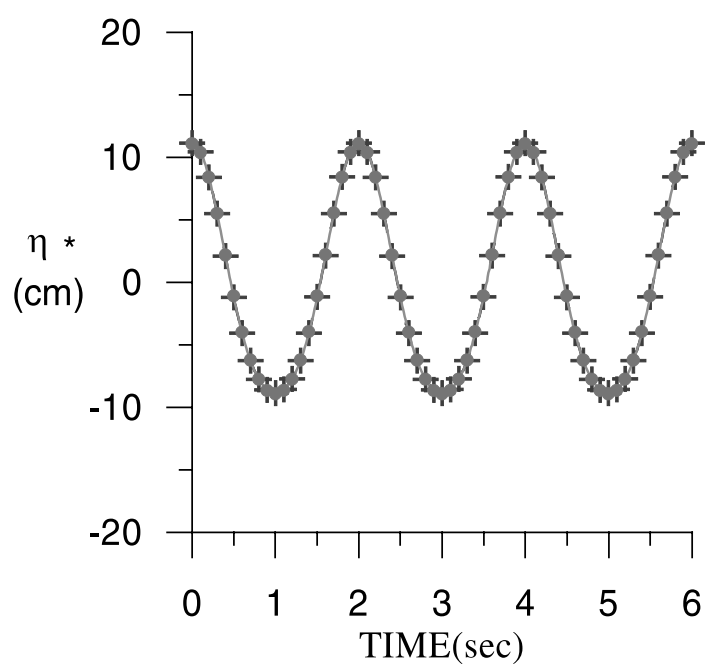

(a)

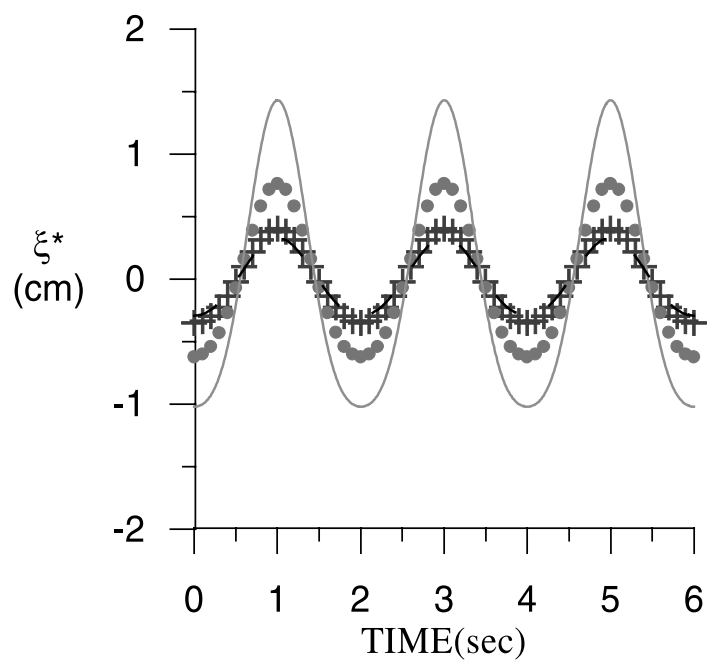

(b)

$$
\begin{aligned}
-\quad \mathrm{U} & =0 \\
+\quad \mathrm{U} & =0.1 \mathrm{~m} / \mathrm{s} \\
\mathrm{U} & =0.5 \mathrm{~m} / \mathrm{s} \\
\mathrm{U} & =1.0 \mathrm{~m} / \mathrm{s}
\end{aligned}
$$

Fig. 10. (a) Free surface profiles and (b) channel bed interface profiles under different currents (wave period $=2 \mathrm{~s}$ ).

\section{Acknowledgements}

This study is sponsored by National Science Council of the Republic of China under grants NSC 882611-E-002-028. 


\section{Appendix A. Coefficients of the solutions of the first and third waves in each order}

A.1. $O\left(\varepsilon_{1}\right)$

$$
\begin{aligned}
& a_{1}=\frac{\mathrm{ie}^{k_{0} h}}{\omega\left(\mathrm{i} q_{1} K_{1}-q_{3} L_{1}\right)-U\left(\mathrm{i} K_{1}-L_{1}\right)}\left[\frac{g k_{0}}{k_{0} U-\omega} \sinh \left(k_{0} h\right)-\left(k_{0} U-\omega\right) \cosh \left(k_{0} h\right)\right], \\
& a_{3}=-L_{1} a_{1}, \\
& L_{1}=\frac{2 \mathrm{i} K_{1}}{1+K_{3}^{2}}, \\
& K_{1}^{2}=1-\Lambda^{2}=1-\frac{k_{1}^{2}}{k_{0}^{2}} \\
& K_{3}^{2}=1-\Psi^{2}=1-\frac{k_{3}^{2}}{k_{0}^{2}} .
\end{aligned}
$$

A.2. $O\left(\varepsilon_{1} \varepsilon_{2}\right)$

$$
\begin{aligned}
& E_{5}=\frac{a_{1} \Lambda^{2}\left(g k_{0}\right)^{3 / 2}}{t_{2}}\left\{\left\lceil k_{0} U+\omega q_{2}+K_{3}^{2}\left(\omega q_{2}-k_{0} U\right)-2 \omega q_{3}\right\rceil r_{2}-2 \mathrm{i} \omega K_{1}\left(q_{1}-q_{2}\right) r_{3}\right\}, \\
& c_{1}=\frac{\mathrm{i}}{N_{1}} \Lambda^{2} a_{1}\left\{1+\frac{1}{t_{2}}\left(1+K_{3}^{2}\right)\left\lceil\left(k_{0} U-\omega\right) \omega C_{0} K_{1}\left(q_{1}-q_{2}\right) r_{1}+g k_{0} r_{2} t_{1}\right\rceil\right\}, \\
& c_{3}=\frac{2}{t_{2}} \Lambda^{2} a_{1}\left[\left(k_{0} U-\omega\right) \omega C_{0} K_{1}\left(q_{1}-q_{2}\right) r_{1}+g k_{0} t_{1} r_{2}\right], \\
& t_{2}=\left(K_{0} U-\omega\right) C_{0} K_{1}\left\lceil-k_{0} U-\omega q_{1}+N_{3}^{2}\left(K_{0} U-\omega q_{1}\right)+2 \omega q_{3}\right\rceil r_{1}-\left\lceil\left(1+K_{3}^{2}\right) r_{2}+2 \mathrm{i} K_{1} r_{3}\right\rceil g k_{0} t_{1}, \\
& t_{1}=\mathrm{e}^{k_{0} h}\left\lfloor\left(k_{0} U-\omega\right)^{2} \cosh \left(k_{0} h\right)-g k_{0} \sinh \left(k_{0} h\right)\right\rfloor, \\
& r_{1}=\mathrm{e}^{k_{0} h}\left\lfloor g k_{0} \cosh \left(k_{0} h\right)-\left(k_{0} U-\omega\right)^{2} \sinh \left(k_{0} h\right)\right\rfloor, \\
& r_{2}=\frac{\left(2 G K_{1}^{2}-\lambda \Lambda^{2}\right)}{(2 G+\lambda) \Lambda^{2}} q_{2}+q_{1}, \\
& r_{3}=\frac{2 \mathrm{i} G K_{3}}{(2 G+\lambda) \Lambda^{2}} q_{2}, \\
& C_{0}=\frac{n_{0} \rho_{0} g}{k_{0} K \Lambda^{2}}, \\
& q_{2}=1-n_{0}+\alpha_{2} n_{0} .
\end{aligned}
$$


A.3. $O\left(\varepsilon_{1}^{2}\right)$

$$
\begin{aligned}
& E_{3}=\frac{f_{13} f_{22} f_{32}+f_{12} f_{23} f_{33}-f_{12} f_{24} f_{32}-f_{13} f_{23} f_{31}}{f_{11} f_{22} f_{32}-f_{12} f_{21} f_{32}-f_{11} f_{23} f_{31}}, \\
& E_{4}=\frac{-2}{g k_{0}}\left(k_{0} U-\omega\right)^{2} E_{3}+\frac{3 \mathrm{i} \sqrt{g k_{0}}}{2\left(k_{0} U-\omega\right)}-\frac{\mathrm{i}}{2} \frac{1}{\left(g k_{0}\right)^{3 / 2}}\left(k_{0} U-3 \omega\right)\left(k_{0} U-\omega\right)^{2}, \\
& b_{1}=\frac{1}{f_{12}}\left(f_{13}-f_{11} E_{3}\right), \\
& b_{3}=\frac{1}{f_{32}}\left(f_{33}-f_{31} b_{1}\right) \text {, } \\
& f_{11}=\frac{-2 \mathrm{i}}{\sqrt{g k_{0}}}\left(k_{0} U-\omega\right)\left[\cosh \left(2 k_{0} h\right)-\frac{2}{g k_{0}}\left(k_{0} U-\omega\right)^{2} \sinh \left(2 k_{0} h\right)\right], \\
& f_{12}=-\frac{k_{0} K \Lambda^{2} \tilde{q}_{1}}{\mathrm{e}^{k_{0} h} n_{0} \rho_{0} g} \\
& f_{13}=-\left[3-\frac{1}{\left(g k_{0}\right)^{2}}\left(k_{0} U-3 \omega\right)\left(k_{0} U-\omega\right)^{3}\right] \sinh \left(2 k_{0} h\right)+\frac{\left(g k_{0}\right)^{2}-\left(k_{0} U-\omega\right)^{4}}{2 g k_{0}\left(k_{0} U-\omega\right)^{2}}+\mathrm{e}^{-k_{0} h}\left(a_{1} K_{1}-\mathrm{i} a_{3}\right) \\
& \times\left[\sinh \left(k_{0} h\right)-\frac{1}{g k_{0}}\left(k_{0} U-\omega\right)^{2} \cosh \left(k_{0} h\right)\right]+\frac{k_{0} K \Lambda^{2}}{\mathrm{e}^{2 k_{0} h} n_{0} \rho_{0} g}\left(a_{1} K_{1}-\mathrm{i} a_{3}\right) a_{1} q_{1} K_{1}, \\
& f_{21}=\mathrm{e}^{k_{0} h}\left[\frac{4}{g k_{0}}\left(k_{0} U-\omega\right)^{2} \cosh \left(2 k_{0} h\right)-2 \sinh \left(2 k_{0} h\right)\right] \text {, } \\
& f_{22}=\frac{2 \mathrm{i}}{\sqrt{g k_{0}}}\left(\tilde{q}_{1} \omega-k_{0} U\right) M_{1}, \\
& f_{23}=\frac{4}{\sqrt{g k_{0}}}\left(\tilde{q}_{3} \omega-k_{0} U\right), \\
& f_{24}=\mathrm{e}^{k_{0} h} \cosh \left(2 k_{0} h\right)\left[\frac{3 \mathrm{i} \sqrt{g k_{0}}}{k_{0} U-\omega}-\frac{\mathrm{i}}{\left(g k_{0}\right)^{3 / 2}}\left(k_{0} U-3 \omega\right)\left(k_{0} U-\omega\right)^{2}\right]-\frac{2 \mathrm{i} \sqrt{g k_{0}}}{k_{0} U-\omega}\left(a_{1} K_{1}-\mathrm{i} a_{3}\right) \\
& \times\left[\cosh \left(k_{0} h\right)-\frac{1}{g k_{0}}\left(k_{0} U-\omega\right)^{2} \sinh \left(k_{0} h\right)\right]-\frac{\mathrm{e}^{-k_{0} h}}{\sqrt{g k_{0}}}\left(a_{1} K_{1}-\mathrm{i} a_{3}\right)\left\lfloor\mathrm{i} a_{1} q_{1} \omega\left(1+K_{1}^{2}\right)\right. \\
& \left.+2 a_{3} q_{3} K_{3} \omega-k_{0} U\left(\mathrm{i} a_{1} K_{1}^{2}+a_{3} K_{3}\right)\right\rfloor, \\
& f_{31}=4 \mathrm{ie}^{k_{0} h} G M_{1}, \\
& f_{32}=\mathrm{e}^{k_{0} h} G\left(M_{3}+4\right), \\
& f_{33}=-\left(a_{1} K_{1}-\mathrm{i} a_{3}\right)\left\lfloor\mathrm{i}\left(2 G+\lambda \Lambda^{2}+2 G K_{1}^{2}\right) a_{1}+G K_{3}\left(K_{3}^{2}+3\right) a_{3}\right\rfloor,
\end{aligned}
$$




$$
\begin{aligned}
& M_{1}^{2}=4-\frac{\tilde{k}_{1}^{2}}{k_{0}^{2}}, \\
& M_{3}^{2}=4-\frac{\tilde{k}_{3}^{2}}{k_{0}^{2}} .
\end{aligned}
$$

\section{References}

Anderson, A.G., 1953. The characteristics of sediment waves formed by flow in open channels. Proceedings of the Third Midwestern Conference in Fluid Mechanics, Minneapolis, March, pp. 379-395.

Bagnold, R.A., 1936. The movement of the desert sand. Proc. Roy. Soc. London A 892, 157, pp. 594-620.

Biot, M.A., 1956. Theory of propagation elastic waves in a fluid saturated porous solid. I. Low-frequency range. JASA $28,168-178$.

Biot, M.A., 1962. Mechanics of deformation and acoustic propagation in porous media. J. Appl. Phys. 33 (4), $1482-1498$.

Chen, T.W., Huang, L.H., Song, C.H., 1997. Dynamic response of poroelastic bed to nonlinear water waves. J. Engng. Mech. ASCE 123 (10), 1041-1049.

Darwin, G.H., 1883-1884. On the Formation of Ripple-Marks. Proc. Roy. Soc., London.

Exner, F.M., 1925. Über die wechselwirkung zwischen wasser und geschiebe in flussen. Sitzungsberichte der Akademie der Wissenschaften, Wein, Heft 3 and 4.

Fenton, J.D., 1985. A fifth-order Stokes theory for steady waves. Wtrwy. Port Coast. Oc. Engng. ASCE 111, $216-234$.

Huang, L.H., Chiang, Y.L., 1998. Bed form formation in alluvial channels. Seventh Int. Symp. River Sedimentation, Hong Kong.

Huang, L.H., Chwang, A.T., 1990. Trapping and absorption of sound waves. II. A sphere covered with a porous layer. Wave Motion $12,401-414$.

Huang, L.H., Song, C.H., 1993. Dynamic response of poroelastic bed to water waves. J. Hydraul. Engng. ASCE 119, $1003-1020$.

Kennedy, J.F., 1963. The mechanics of dunes and antidunes in erodible-bed channels. J. Fluid Mech. 16, 521-544.

Liu, L.F., 1973. Damping of water waves over porous bed. J. Hydraul. Div. ASCE 99 (12), 2263-2271.

Mei, C.C., 1983. The Applied Dynamics of Ocean Waves. Wiley, New York.

Mei, C.C., Foda, M.A., 1981. Wave-induced responses in a fluid filled poroelastic solid with a free surface a boundary layer theory. Geophys. J. R. Astr. Soc. 66, 597-631.

Press, W.H., Teukolsky, S.A., Vetterling, W.T., Flannery, B.P., 1992. Numerical recipes in Fortran, the art of scientific computing, third ed. Cambridge University Press, Cambridge, pp. 550-554 (Chapter 15.1).

Putnam, J.A., 1949. Loss of wave energy due to percolation in a permeable sea-bottom. Trans. Am. Geophys. Union $30,349-356$.

Reid, R.O., Kajiura, K., 1957. On the damping of gravity waves over a permeable sea bed. Trans. Am. Geophys. Union 30, $662-666$.

Sleath, J.F.A., 1970. Wave induced pressure in bed of sand. J. Hydraul. Div. ASCE 96, 367-378. 